\title{
Energy efficiency maximization of full-duplex two-way relay-assisted device-to-device communications underlaying cellular networks
}

\author{
Yiliang Chang ${ }^{1}$, Hongbin Chen ${ }^{1,2^{*}}$ (D) and Feng Zhao ${ }^{1}$
}

\begin{abstract}
With the substantial progress in self-interference (SI) cancelation, the full-duplex (FD) technique, which allows the communication user to transmit and receive signals over the same frequency band simultaneously, enables a significant enhancement of spectral efficiency (SE) in comparison with the traditional half-duplex (HD) technique. Recently, relay-assisted device-to-device (D2D) communications underlaying cellular networks have aroused a great deal of research interests due to its high SE. For the new meaningful paradigm of the combination of the FD and the amplify-and-forward (AF) relay-assisted D2D communications, analyzing the SE and energy efficiency (EE) is crucial, which have not been investigated in the existing works. In this paper, we focus on the EE of the FD twoway (FDTW) relay-assisted D2D communications with uplink channel reuse by considering the residual SI at the D2D users and compare it with the HD counterpart. Our goal is to find the optimal transmission powers and amplification gain to maximize the system EE while guaranteeing SE requirements and maximum transmission power constraints. A new two-tier alternative iteration optimization algorithm is proposed to solve the optimization problem. Simulation results show that (1) the results obtained by the proposed algorithm is very close to those obtained by the exclusive searching method, (2) smaller residual power of SI leads to better performance of EE and SE, (3) the SE obtained by FDTW relay-assisted D2D networks is higher than the SE obtained by the HD counterpart, and (4) the EE comparison of FDTW relay-assisted D2D networks and its HD counterpart depends on the residual power of SI. The EE obtained by FDTW relay-assisted D2D is higher than the EE obtained by HD counterpart only when the residual power of $\mathrm{SI}$ is sufficiently small.
\end{abstract}

Keywords: Relay-assisted device-to-device (D2D) communications, Full-duplex (FD), Self-interference (SI), Energy efficiency (EE), Spectral efficiency (SE)

\section{Introduction}

It is expected that mobile data traffic will increase a thousand-fold over the next decade which will be driven by the expected 50 billion mobile devices connected to the cloud, anywhere and anytime. With a rapid increase in the number of connected devices, some challenges for next 5G networks appear which will be responded by increasing capacity and by improving energy efficiency (EE), coverage, spectrum utilization, and so on [1]. As

\footnotetext{
* Correspondence: chbscut@guet.edu.cn

${ }^{1}$ Key Laboratory of Cognitive Radio and Information Processing, Guilin

University of Electronic Technology, Guilin 541004, China

${ }^{2}$ Guangxi Experiment Center of Information Science, Guilin 541004, China
}

the key techniques to address the requirements for $5 \mathrm{G}$ networks, direct device-to-device (D2D) communication and in-band full-duplex (FD) have attracted a great deal of research interests [2-4].

D2D communications allow proximate cellular users to communicate with each other directly under the control of base station (BS) with lower transmit power requirements. The high channel quality of short-range D2D links facilitate high data rates for local services, prolong users' battery lives, and offload heavy traffic of BS [5]. In addition, D2D links can underlay cellular links by reusing the same time and frequency resources; thus, the spectral efficiency (SE) of cellular networks can be 
improved obviously. However, the introduction of D2D communications into cellular networks poses new challenge in the resource allocation design due to cochannel interference caused by spectrum reuse, which has marked impacts on the performance of communication reliability and network throughput. Thus, effective power allocation is required to alleviate the interference problem. Besides, another efficient way to reduce the impact of interference is providing a relay user between D2D pair [6]. We refer to this as relay-assisted D2D communications which can be an efficient approach to provide a better Quality of Service (QoS) and lower transmission powers for communication between distant D2D users.

Relay communication, in which the relay forwards the signal received by a source to a destination, has been envisaged as a spectral- and energy-efficient technology for cellular networks [7]. Based on the directions of signal transmission, relaying systems can be classified into three transmission types (i.e., one-way, two-way, and multiway). Compared with one-way relaying, two-way relaying can achieve higher $\mathrm{SE}$, which allows a relay user to simultaneously communicate with two end users. Existing studies also show that the two-way relayassisted D2D communications have significant improvement on system performance (e.g., outage probability, system sum rate) $[8,9]$. Thus, with the assistance of two-way relaying, the performance advantage of D2D communications can be further improved. Multiway relaying, in which multiple users exchange information with the assistance of a relay, can achieve better performance and flexibility than two-way relaying. However, its protocol and encoder design are rather complicated, making it a potential choice for relay-assisted D2D communications in the future.

On the other hand, the in-band FD transmission, which allows transmitting and receiving at the same frequency and the same time, enables an enormous enhancement of SE than half-duplex (HD) [10-12]. The main limitation impacting FD transmission is the strong self-interference (SI) signal imposed by the transmit antenna on the receive antenna within the same transceiver. Both academia and industry reached consensus that SI cancelation would play the most pivotal role in implementing FD communication systems. There are usually two main categories: passive suppression (PS) and active cancelation [13]. Therein, the active cancelation also includes analog cancelation (AC) and digital cancelation (DC). Since none of the individual cancelation techniques is capable of satisfying the system requirements in terms of the attainable SI cancelation capability, a high-capability cancelation scheme by combining the active and passive techniques is necessarily developed. Among all the SI cancelation techniques, the
PS has an important position. The primary advantage to performing PS in the propagation domain is that the downstream receiver hardware can process signals more accurately with a large dynamic range [10]. In addition, DC techniques can be performed in the digital domain, which are the lowest-complexity active SI cancelation techniques in FD systems $[10,14]$. As the design of AC usually requires a complex and large-size hardware circuits with respect to mobile devices [11, 14, 15], a combination of the latest PS and DC (referred to as PSDC) techniques will be an appropriate way to implement the mobile FD system $[13,14]$.

Hence, it can be practically beneficial applying the FD techniques into two-way relay-assisted D2D communications with certain SI cancelation where the relay user and D2D users can transmit and receive signals simultaneously, and thus, the SE gets further improved. Since FD transmission possesses strong SI, it generally consumes more power than HD. Therefore, there is an urgent demand for maintaining high system throughput while limiting energy consumption. EE, defined as the ratio of throughput to power consumption, is an important measure of green communication solutions [7]. It is urgent and interesting to investigate the EE of FD twoway (FDTW) relay-assisted D2D communications underlaying cellular networks.

\subsection{Related work}

In the existing literature, many works have been done on the EE of D2D communications underlaying cellular networks. For example, the EE of D2D communications without channel reuse was investigated in [16]. The authors showed the EE improvement with the deployment of D2D communications in heterogeneous networks (HetNets) compared with the full small-cell deployment, thus provided a greener alternative to cellular network deployment. Correspondingly, [17] and [18] presented the analysis of the EE of D2D systems with cellular channel reuse. Therein, [17] revealed the tradeoff between EE and delay in D2D communications, where stochastic traffic arrivals and time-varying channel conditions were both considered. Given the SE requirement and maximum transmission power constraints, the authors in [18] investigated the EE and SE, in which each user is self-interested and wants to maximize its own EE. Inspired by the performance gain of a combination of relay and D2D communications, the authors in [19] extended radio resource management algorithms into one-way relay-assisted D2D communications to balance SE and EE while considering mode selection and resource allocation constraints. In [6], the maximum achievable transmission capacity was studied in one-way relay-assisted D2D communications while guaranteeing the outage probability of both cellular and D2D links. 
For a higher performance improvement, two-way relaying with network coding technique which can improve transmission efficiency will be a promising choice in D2D communications. The authors in [20] demonstrated the performance gain of D2D communications assisted by two-way relaying over Rayleigh fading channels. The achievable rate of different two-way transmission schemes of relay-assisted D2D communications were presented in [9], while considering the interference due to spectrum sharing. Compared with the traditional cellular communications through BS, the EE and SE of two-way relay-assisted D2D communications were studies in [21]. The optimal user equipment (UE) transmission powers to maximize the EE were also derived. However, there is little work addressing the EE in FDTW relay-assisted D2D communications underlaying cellular networks, which is the focus of this paper.

\subsection{Summary}

In this paper, we provide a comprehensive EE analysis of FDTW relay-assisted D2D communications underlaying cellular networks, in comparison to its HD counterpart. The contributions of this paper are summarized as follows.

- To the best of our knowledge, this is the first work to investigate the EE of FDTW relay-assisted D2D communications underlaying cellular networks. Moreover, the comparison between the FDTW relay-assisted D2D network and the HD two-way (HDTW) relay-assisted D2D network in terms of SE and EE is made. Finally, we demonstrate that the proposed two-tier alternative iteration optimization algorithm can converge very quickly.

- From the perspective of practical implementation, SI cannot be fully canceled due to technical challenges. Therefore, we consider remaining SI after cancelation at the FD users in a more reasonable and relatively tractable way to study EE and SE in the FDTW relay-assisted D2D communications.

- The joint optimization problem is non-convex. To solve this issue, the primal optimization problem is first decomposed into two subproblems, and then, we solve the two subproblems alternatively. For the first subproblem, we prove it is convex and then solve it by the bisection method. For the second subproblem, we decomposed it into two subsubproblems again, and then, we solve the two sub-subproblems alternatively. For the first subsubproblem, we transform it into concave function by using the nonlinear fractional programming, which can be effectively solved by the Dinkelbach method [22]. For the second sub-subproblem, we first prove it is convex and then solve it by the bisection method.
- A full range of power consumption sources is considered in the power consumption model. In particular, the power consumed by SI cancelation is considered. The impact of imperfect SI cancelation is also considered in the analysis.

The rest of this paper is organized as follows. Section 2 describes the system model and optimization problem for FDTW relay-assisted D2D communications underlaying cellular networks. The elaborate solving process of the optimization problem is presented in Section 3. In Section 4, simulation results are presented. Finally, some concluding remarks are given in Section 5.

\section{System model}

In this paper, we consider the FDTW relay-assisted D2D communications underlaying the cellular networks where the uplink radio resource is shared by the D2D link as depicted in Fig. 1. The cellular link consists of a cellular user equipment (CUE) and a receiver base station (BS), while the D2D link consists of a D2D pair (including source user equipment (SUE) and destination user equipment (DUE)) and a relay user equipment (RUE). In the FDTW relay-assisted D2D link, SUE intends to exchange information with DUE under the assistance of RUE, where SUE and DUE both transmit their signals to RUE, and at the same time, RUE broadcasts the previous received signals to SUE and DUE. Each user of the D2D link operates in the FD mode (with one antenna dedicated to transmission and another to reception, in order to increase the isolation of the SI [23]), which can double the spectral resource utilization potentially, and the CUE operates in traditional cellular networks with a single antenna. There is no direct link between SUE and DUE (e.g., due to the shadowing effect or the poor direct link condition).

D2D transmissions will cause interference to the BS receiver, and D2D receivers also suffer interference from the cellular uplink transmission. Moreover, the users in FD D2D link transmit and receive at the same time and frequency, causing SI to the receivers from its transmitters. It can be suppressed by the methods in [13, 14], which is beyond the scope of this paper. However, due to the practical constraint, the residual SI after interference suppression still exists and limits the performance of FDTW relay-assisted D2D network, which becomes the main concern in this paper. Hence, in order to analyze the effect of SI on system performance, we assume the SI in the FDTW relay-assisted D2D communications can be reduced but cannot be completely eliminated by SI cancelation techniques. The existence of SI will make the optimization problem formulation complicated as shown later. Let $\rho$ denotes the average residual power of SI after cancelation at each user in 


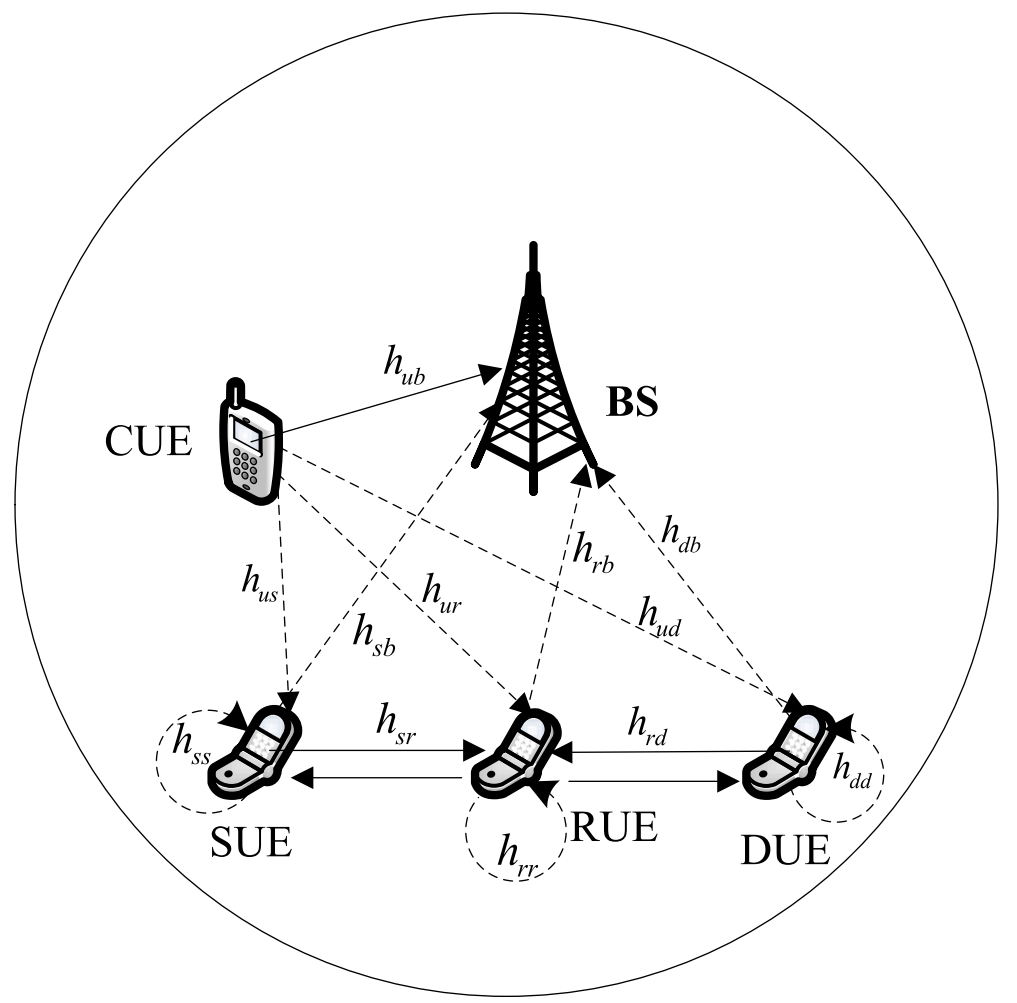

Fig. 1 FDTW relay-assisted D2D communications

D2D link. Based on the experimental results in [23], the residual SI power model general includes two cases: the complicated case that SI power increase linearly with the transmission power [24], and the other one in which the SI power is a constant and not a function of the transmission power [25]. Similar to our previous work in [26], we consider the second case that SI power is modeled as an invariable value. There are two main reasons for us to employ this model. One is that if $\rho$ is regarded as a function of the transmission power of the D2D users, both the primal problem (16) and the transformed subproblem (P2) (defined in Sections 2 and 3.2, respectively) may become non-convex. The second reason is that the power of SI can be controlled in a range after cancelation [27]. Thus, it is reasonable to regard $\rho$ to be fixed. The issue that $\rho$ may be associated with the transmission power of D2D users will be considered in our future work. The SI channel coefficients at SUE, RUE, and DUE are denoted by $h_{s s}, h_{r n}$ and $h_{d d}$ respectively, which are generally modeled by the zero-mean circularly symmetric complex Gaussian (CSCG) random variables.

Assume that the channel coefficient between SUE and RUE and the channel coefficient between RUE and DUE are both reciprocal and denoted by $h_{s r}$ and $h_{r d}$, respectively. Herein, the subscripts $s, r, d$ represent SUE, RUE, and DUE, respectively. At the time instant $t$, CUE transmits its symbol to BS, and SUE and DUE transmit their respective symbols to RUE, respectively. Simultaneously, RUE broadcasts another symbol $r(t)$ to SUE and DUE. We consider quasi-static fading in which the channel coefficients are constant within one defined frame but may change independently from one frame to another. Therefore, at the time instant $t$, the received signal at RUE can be expressed as

$$
\begin{aligned}
y_{r}(t) & =\sqrt{P_{s}} h_{s r} x_{s}(t)+\sqrt{P_{d}} h_{r d} x_{d}(t)+\sqrt{\rho_{r}} h_{r r} r(t) \\
& +\sqrt{P_{u}} h_{u r} x_{u}(t)+N_{r}(t)
\end{aligned}
$$

where $x_{s}(t), x_{d}(t)$, and $x_{u}(t)$ are the transmitted signals of SUE, DUE, and CUE, respectively, with $E\left\{\left|x_{s}(t)\right|^{2}\right\}$ $=E\left\{\left|x_{d}(t)\right|^{2}\right\}=E\left\{\left|x_{u}(t)\right|^{2}\right\}=1$. Here, $E\{x\}$ defines the expectation of the random variable $x$, and $P_{s}, P_{d}$, and $P_{u}$ are the transmission power of SUE, DUE, and CUE, respectively. $\rho_{r}$ is the residual SI power of RUE which depends on the amount of SI cancelation, and $h_{u r}$ denotes the channel coefficient between the CUE and RUE. $N_{r}(t)$ is additive white Gaussian noise (AWGN) with zero mean and variance $\sigma^{2}$. The expression (1) reveals that for the HDTW relay-assisted D2D communications, the SI is zero, and thus $y_{r}(t)$ has no relationship with its own transmitted symbol $r(t)$. However, for the FDTW relay-assisted D2D communications, $y_{r}(t)$ is decided by $r(t)$, due to the existence of residual SI. 
RUE broadcasts the received signal from both SUE and DUE at the time instant $t-1$ with the amplifyand-forward (AF) protocol, namely, $r(t)=y_{r}(t-1)$. To meet the transmission power constraint of RUE, the average power of $r(t)$ is normalized to 1 . Hence, with network coding technique [28], each user knows its transmitted signal, the received signals after subtracting interference at SUE and DUE are, respectively, given by

$$
\begin{aligned}
y_{s}(t)= & \sqrt{\beta} h_{s r} r(t)+\sqrt{P_{u}} h_{u s} x_{u}(t)+\sqrt{\rho_{s}} h_{s s} x_{s}(t)+N_{s}(t) \\
= & \sqrt{\beta P_{d}} h_{s r} h_{r d} x_{d}(t-1)+\sqrt{\beta \rho_{r}} h_{s r} h_{r r} r(t-1) \\
& +\sqrt{\beta P_{u}} h_{s r} h_{u r} x_{u}(t-1)+\sqrt{\beta} h_{s r} N_{r}(t-1) \\
& +\sqrt{P_{u}} h_{u s} x_{u}(t)+\sqrt{\rho_{s}} h_{s s} x_{s}(t)+N_{s}(t)
\end{aligned}
$$$$
y_{d}(t)=\sqrt{\beta} h_{r d} r(t)+\sqrt{P_{u}} h_{u d} x_{u}(t)+\sqrt{\rho_{d}} h_{d d} x_{d}(t)+N_{d}(t)
$$$$
=\sqrt{\beta P_{s}} h_{s r} h_{r d} x_{s}(t-1)+\sqrt{\beta \rho_{r}} h_{r d} h_{r r} r(t-1)
$$$$
+\sqrt{\beta P_{u}} h_{r d} h_{u r} x_{u}(t-1)+\sqrt{\beta} h_{r d} N_{r}(t-1)
$$$$
+\sqrt{P_{u}} h_{u d} x_{u}(t)+\sqrt{\rho_{d}} h_{d d} x_{d}(t)+N_{d}(t)
$$

where $h_{u s}$ and $h_{u d}$ denote the channel coefficient between CUE and SUE and the channel coefficient between CUE and DUE, respectively. $\beta$ is the amplification gain of RUE, which should satisfy the constraint $0 \leq \beta \leq \bar{\beta}$ with $\bar{\beta}=P_{r \max } /\left(P_{s}\left|h_{s r}\right|^{2}+P_{d}\left|h_{r d}\right|^{2}+\rho_{r}\left|h_{r r}\right|^{2}+P_{u}\left|h_{u r}\right|^{2}+\sigma^{2}\right)$, and $P_{r \max }$ is the maximum allowed transmission power of RUE. $\rho_{s}$ and $\rho_{d}$ are the residual SI power of SUE and DUE which depend on the amount of SI cancelation, respectively. $N_{s}(t)$ and $N_{d}(t)$ are AWGN with zero mean and variance $\sigma^{2}$.

At the same time, cellular uplink transmission will suffer interference both from the D2D pair and the RUE. Therefore, at the time instant $t$, the received signal at BS can be expressed as

$$
\begin{aligned}
y_{u}(t)= & \sqrt{P_{u}} h_{u b} x_{u}(t)+\sqrt{P_{s}} h_{s b} x_{s}(t)+\sqrt{P_{d}} h_{d b} x_{d}(t) \\
& +\sqrt{\beta} h_{r b} r(t)+N_{b}(t) \\
= & \sqrt{P_{u}} h_{u b} x_{u}(t)+\sqrt{P_{s}} h_{s b} x_{s}(t)+\sqrt{P_{d}} h_{d b} x_{d}(t) \\
& +\sqrt{\beta P_{s}} h_{s r} h_{r b} x_{s}(t-1)+\sqrt{\beta P_{d}} h_{r d} h_{r b} x_{d}(t-1) \\
& +\sqrt{\beta \rho_{r}} h_{r b} h_{r r} r(t-1)+\sqrt{\beta P_{u}} h_{r b} h_{u r} x_{u}(t-1) \\
& +\sqrt{\beta} h_{r b} N_{r}(t-1)+N_{b}(t)
\end{aligned}
$$

where $h_{u b}, h_{s b}, h_{r b}$, and $h_{d b}$ denote the channel coefficient between BS and CUE, SUE, RUE, and DUE, respectively. $N_{b}(t)$ is AWGN with zero mean and variance $\sigma^{2}$.

For a more concise expression, the channel gain between the transmitter $i$ and $j$ is denoted as $G_{i j}$ $=\left|h_{i j}\right|^{2}$, where $h_{i j}$ is CSCG random variable with variance $\sigma_{i j}=d_{i j}^{-v}$. Herein, $d_{i j}$ is the normalized distance between nodes $i$ and $j$, and $v$ is the path-loss exponent.

According to formulas (2), (3), and (4), the instantaneous received signal-to-interference-plus-noise ratios (SINRs) at SUE, DUE, and CUE can be respectively calculated as

$$
\gamma_{s}=\frac{\beta P_{d} G_{s r} G_{r d}}{\beta P_{u} G_{s r} G_{u r}+\beta \rho_{r} G_{s r} G_{r r}+\beta G_{s r} \sigma^{2}+P_{u} G_{u s}+\rho_{s} G_{s s}+\sigma^{2}}
$$

$$
\gamma_{d}=\frac{\beta P_{s} G_{s r} G_{r d}}{\beta P_{u} G_{r d} G_{u r}+\beta \rho_{r} G_{r d} G_{r r}+\beta G_{r d} \sigma^{2}+P_{u} G_{u d}+\rho_{d} G_{d d}+\sigma^{2}}
$$

$$
\begin{aligned}
& \gamma_{u}=\frac{P_{u} G_{u b}}{P_{s} G_{s b}+P_{d} G_{d b}+\beta P_{s} G_{s r} G_{r b}+\beta P_{d} G_{r d} G_{r b}+\beta \rho_{r} G_{r b} G_{r r}} \\
& +\beta P_{u} G_{u r} G_{r b}+\beta G_{r b} \sigma^{2}+\sigma^{2}
\end{aligned}
$$

The SE of cellular link and FDTW relay-assisted D2D link are written as

$$
\begin{aligned}
& \eta_{u, S E}\left(P_{s}, P_{d}, \beta, P_{u}\right)=R_{u} \\
& \eta_{d, S E}\left(P_{s}, P_{d}, \beta, P_{u}\right)=R_{s}+R_{d}
\end{aligned}
$$

where $R_{u}=\log _{2}\left(1+\gamma_{u}\right), \quad R_{s}=\log _{2}\left(1+\gamma_{s}\right), \quad$ and $\quad R_{d}=$ $\log _{2}\left(1+\gamma_{d}\right)$.

Hence, the overall system SE which is the sum of cellular link and FDTW relay-assisted D2D link can be given by

$$
\begin{aligned}
\eta_{S E}\left(P_{s}, P_{d}, \beta, P_{u}\right) & =\eta_{u, S E}\left(P_{s}, P_{d}, \beta, P_{u}\right)+\eta_{d, S E}\left(P_{s}, P_{d}, \beta, P_{u}\right) \\
& =R_{u}+R_{s}+R_{d}
\end{aligned}
$$

The power consumption in a general wireless node for communications usually contains two main parts: power amplifier and circuit power [18]. Power amplifier is related to the power amplifier drain efficiency and the transmission power level and is usually modeled as the ratio of the transmission power to the power amplifier drain efficiency [10]. Circuit power consumption is usually considered to be independent of the data rate and is regarded as a constant [29]. We 
consider both power amplifier and circuit power consumption in the transmitters. In addition, to be more practical, the circuit power consumption in the receivers is also considered. In a practical system, the circuit power consumption of BS in uplink is huge that can be supported by various efficient energy sources including renewable energy. Therefore, the EE of BS would be less critical as compared to that in the users since mobile devices are typically hand-held devices with limited battery life and can quickly run out of battery. So, it is reasonable to neglect the circuit power consumption of BS in uplink in the system EE $[17,18]$. In addition, in FD communications, applying PS generally does not consume additional power; however, the power consumed by active DC is nonnegligible. The power consumed by $\mathrm{DC}, P_{\mathrm{DC}}$, is regarded as a constant due to the power consumed by the involved chip components which are not related to the throughput state.

Therefore, the power consumption of cellular link and FDTW relay-assisted D2D link can be respectively expressed as

$$
\begin{gathered}
P_{u, \text { total }}\left(P_{u}\right)=\xi P_{u}+P_{c} \\
\begin{array}{c}
P_{d, \text { total }}\left(P_{s}, P_{d}, \beta, P_{u}\right)=\xi P_{s}+\xi P_{d}+\beta\left(P_{s} G_{s r}+P_{d} G_{r d}+\rho_{r} G_{r r}\right. \\
+ \\
\left.+P_{u} G_{u r}+\sigma^{2}\right)+3 P_{D C}+3 P_{c}
\end{array}
\end{gathered}
$$

where $1 / \xi$ is the power amplifier drain efficiency of the CUE, SUE, and DUE, respectively. $P_{c}$ is the static circuit power.

The EE of cellular link and FDTW relay-assisted D2D link is written as

$$
\begin{aligned}
& \eta_{u, E E}\left(P_{s}, P_{d}, \beta, P_{u}\right)=\frac{W \eta_{u, S E}\left(P_{s}, P_{d}, \beta, P_{u}\right)}{P_{u, \text { total }}\left(P_{u}\right)} \\
& \eta_{d, E E}\left(P_{s}, P_{d}, \beta, P_{u}\right)=\frac{W \eta_{d, S E}\left(P_{s}, P_{d}, \beta, P_{u}\right)}{P_{d, \text { total }}\left(P_{s}, P_{d}, \beta, P_{u}\right)}
\end{aligned}
$$

respectively, where $W$ denotes the channel bandwidth. Since powers of different users cannot be shared and so are their throughput and $\mathrm{EE}$, a novel definition of system $E E$ based on summation of $E E$ of all transmitters rather than the ratio of sum network throughput to sum network power consumption was proposed in [30]. Inspired of this novel definition, we define the system $\mathrm{EE}$ of the FDTW relay-assisted D2D communications underlaying cellular networks as the sum of the EE of cellular link and FDTW relay-assisted D2D link. Therefore, the EE of the whole system can be expressed as

$$
\eta_{E E}=\eta_{d, E E}\left(P_{s}, P_{d}, \beta, P_{u}\right)+\eta_{u, E E}\left(P_{s}, P_{d}, \beta, P_{u}\right)
$$

Our objective is to find the optimal transmission powers $P_{s}, P_{d}$, and $P_{u}$ and the optimal relay amplification gain $\beta$ to maximize $\eta_{E E}$ while keeping $\eta_{d \text {,SE }}$ and $\eta_{u \text {,SE }}$ above the thresholds and satisfying the maximum power constraints. The optimization problem can be formulated as

$$
\begin{gathered}
\max _{\left\{P_{s}, P_{d}, \beta, P P_{u}\right\}} \eta_{\mathrm{EE}} \\
\text { s.t. }\left\{\begin{array}{l}
\eta_{u, \mathrm{SE}} \geq \bar{\eta}_{u, \mathrm{SE}} \\
\eta_{d, S E} \geq \bar{\eta}_{d, \mathrm{SE}} \\
0 \leq P_{u} \leq P_{u, \max } \\
0 \leq P_{s} \leq P_{s, \max } \\
0 \leq P_{d} \leq P_{d, \max } \\
0 \leq \beta \leq \bar{\beta}
\end{array}\right.
\end{gathered}
$$

where $\bar{\eta}_{u \text { SE }}$ and $\bar{\eta}_{d, S E}$ denote the minimum SE requirements of the cellular link and the D2D link and $\bar{\eta}_{u, \mathrm{SE}}=R_{u, \min }$ and $\bar{\eta}_{d \mathrm{SE}}=R_{s, \text { min }}+R_{d, \text { min }}$, here $R_{u, \text { min }}, R_{s, \text { min }}$, and $R_{d, \text { min }}$ are the minimum data rate requirements of CUE, SUE, and DUE, respectively. $P_{u, \max }, P_{s, \max }$, and $P_{d, \max }$ are the maximum allowed transmission power of the CUE, SUE, and DUE, respectively.

\section{Energy efficiency maximization problem}

In this section, we formulate the EE optimization problem for FDTW relay-assisted D2D communications underlaying the cellular networks. Specifically, we will seek the optimal transmission powers $P_{s}, P_{d}, P_{u}$, and the optimal relay amplification gain $\beta$ to maximize EE while keeping SE above a threshold and guaranteeing transmission power constraints. Unfortunately, joint optimization over $P_{s}, P_{d}, P_{u}$, and $\beta$ is very hard to be found due to that $\eta_{\mathrm{EE}}$ is not concave in $P_{s}, P_{d}, P_{u}$, and $\beta$ jointly. So, it cannot be solved by the general convex optimization methods. To overcome this difficulty, a new two-tier alternative iteration optimization algorithm is proposed in this section. The basic idea is to first optimize the EE of the cellular link by the bisection method and the FDTW relay-assisted D2D link with iterative optimization algorithm, respectively, and then alternately iterate the cellular link and the FDTW relay-assisted D2D link until convergence to produce the optimal system EE.

\subsection{EE maximization of cellular link with fixed $P_{s,} P_{d \prime}$ and $\beta$}

In the EE optimization problem of the cellular link, given fixed $P_{s}, P_{d}$, and $\beta$, our objective is to seek the optimal transmission power $P_{u}$ of CUE to maximize $\eta_{u, \mathrm{EE}}$ while keeping $\eta_{u \text {,SE }}$ above a threshold and satisfying the maximum power constraint. The suboptimization problem can be formulated as 


$$
\begin{array}{ll}
\max _{P_{u}} & \eta_{u, \mathrm{EE}} \\
\text { s.t. } & C 1: \eta_{u, \mathrm{SE}} \geq \bar{\eta}_{u, \mathrm{SE}} \\
& C 2: 0 \leq P_{u} \leq P_{u, \max }
\end{array}
$$

It is easy to prove that $\eta_{u, \mathrm{SE}}$ is a monotonically increasing function of $P_{u} \in[0,+\infty)$, and according to the restricted conditions of $\eta_{u, \text { SE }}$ (i.e., $\eta_{u, S E} \geq 0, \eta_{u, S E}$ $\left.\geq \bar{\eta}_{u, S E}\right)$, we have $P_{u} \in\left\{P_{u} \mid P_{u} \in\left(\widetilde{P}_{u},+\infty\right)\right\}$, where $\breve{P_{u}}=G_{2}\left(2^{\bar{\eta}_{u, S E}}-1\right) / G_{u b}-\left(2^{\bar{\eta}_{u, S E}}-1\right) G_{1}, G_{1}=\beta G_{u r} G_{r b}$, and $G 2=P_{s} G_{s b}+P_{d} G_{d b}+\beta\left(P_{s} G_{s r} G_{r b}+P_{d} G_{r d} G_{r b}+\rho_{r} G_{r b} G_{r r}\right.$ $\left.+G_{r b} \sigma^{2}\right)+\sigma^{2}$.

Since $P_{u} \in\left[0, P_{u, \max }\right]$ and $P_{u} \in\left\{P_{u} \mid P_{u} \in\left(\widetilde{P}_{u},+\infty\right)\right\}$, the feasible region of the subproblem $(\mathrm{P} 1)$ can be rewritten as $P_{u} \in\left[\breve{P}_{u}, P_{u, \max }\right]$.

3.1.0.1 Theorem 1 Given $P_{s}, P_{d}, \beta \in[0,+\infty), \quad \eta_{u, \mathrm{EE}}$ is strictly quasiconcave in $P_{u}$ for $P_{u} \in[0,+\infty)$.

3.1.0.2 Proof Refer to Appendix 1.

From Theorem 1, there are only three cases for the curve

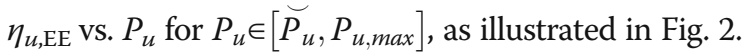

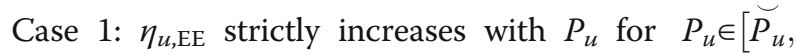
$\left.P_{u, \text { max }}\right]$, if $\left.\frac{d \eta_{u, \mathrm{EE}}}{d P_{u}}\right|_{P_{u}=P_{u, \max }} \geq 0$, where $\frac{d \eta_{u, \mathrm{EE}}}{d P_{u}}$ is given by (29) in Appendix 1. In this case, the optimal solution to the subproblem (P1) is achieved at $\hat{P}_{u}=P_{u, \max }$.

Case 2: $\eta_{u, \mathrm{EE}}$ strictly decreases with $P_{u}$ for $P_{u} \in\left[\widetilde{P_{u}}\right.$, $\left.P_{u, \text { max }}\right]$, if $\left.\frac{d \eta_{u, \mathrm{EE}}}{d P_{u}}\right|_{P_{u}=\widetilde{P}_{u}} \leq 0$. The optimal solution to the subproblem (P1) is achieved at $\hat{P}_{u}=\widetilde{P}_{u}$.

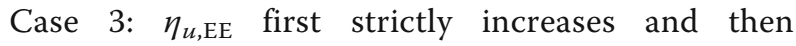
strictly decreases with $P_{u}$ for $P_{u} \in\left[\widetilde{P}_{u}, P_{u, \max }\right]$, if $\left.\frac{d \eta_{u, \mathrm{EE}}}{d P_{u}}\right|_{P_{u}=P_{u, \max }}<0$ and $\left.\frac{d \eta_{u, \mathrm{EE}}}{d P_{u}}\right|_{P_{u}=\breve{P_{u}}}>0$. The optimal solution to the subproblem (P1) is achieved at $\hat{P}_{u}=P_{u}{ }^{*}$, where $P_{u}{ }^{*}$ is the point at which $\eta_{u, \mathrm{EE}}$ reaches its maximum for $P_{u} \in[0,+\infty)$ and is obtained by solving the equation $\frac{d \eta_{u, \mathrm{EE}}}{d P_{u}}=0$, as shown in Appendix 1. The exact expression of $P_{u}{ }^{*}$ is not easily obtained and can be found by the numerical methods, such as the bisection method.

\subsection{EE maximization of FDTW relay-assisted D2D link with a fixed $P_{u}$}

In the EE optimization problem of FDTW relayassisted D2D link, given a fixed $P_{u}$, our objective is to seek the optimal transmission powers $P_{s}, P_{d}$ and the optimal relay amplification gain $\beta$ to maximize $\eta_{d, \mathrm{EE}}$ while keeping $\eta_{d \text {,SE }}$ above a threshold and satisfying the maximum power constraints. The sub-optimization problem can be formulated as

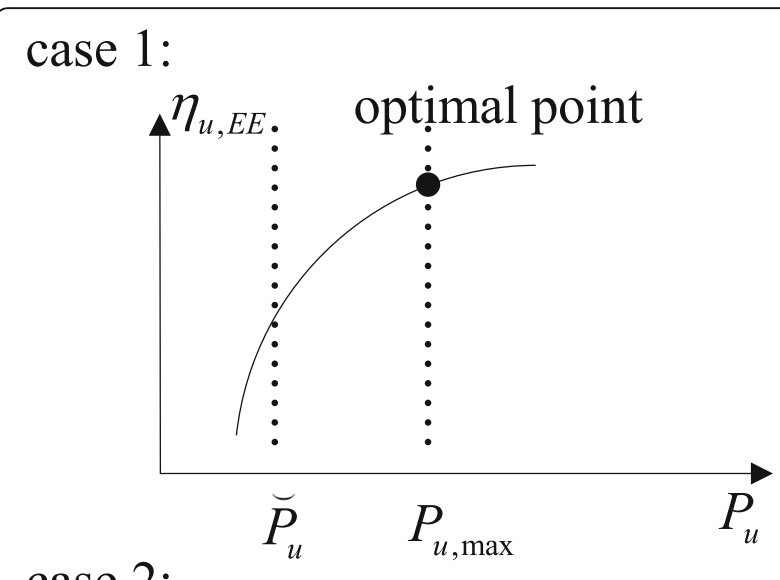

case 2:

case 3 :
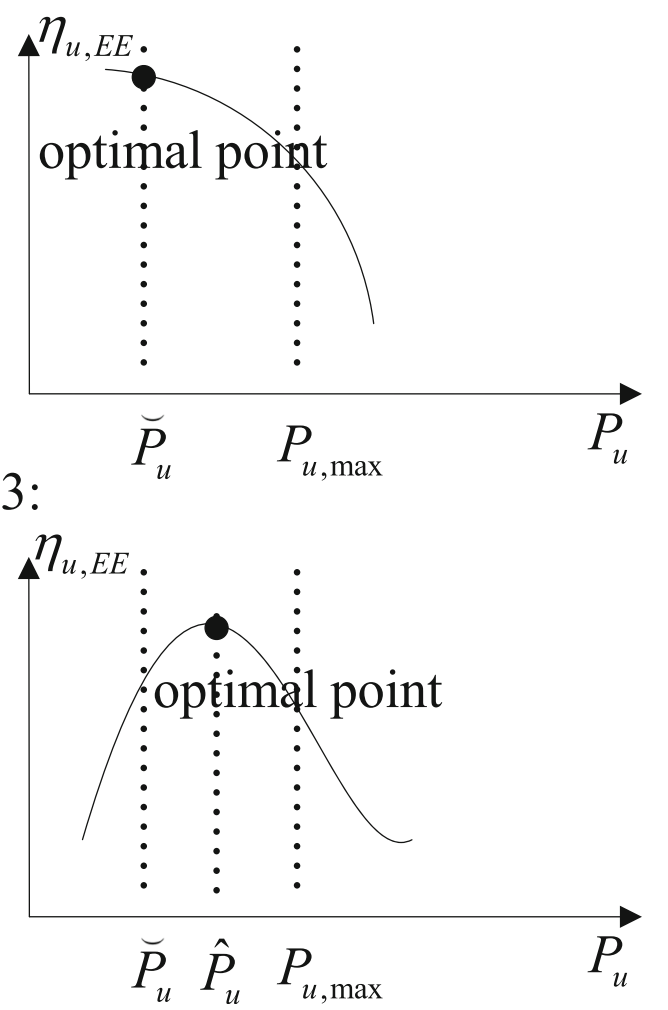

Fig. 2 Three cases of the $\eta_{u, E E}$ versus $P_{u}$ curve

$$
\begin{array}{ll}
\max _{P_{s}, P_{d}, \beta} & \eta_{d, E E} \\
\text { s.t. } & C 3: \eta_{d, S E} \geq \bar{\eta}_{d, S E} \\
& C 4: 0 \leq P_{s} \leq P_{s, \max } \\
& C 5: 0 \leq P_{d} \leq P_{d, \max } \\
& C 6: 0 \leq \beta \leq \bar{\beta}
\end{array}
$$

In the sub-optimization problem (P2), both $\eta_{d \text {,EE }}$ and $\eta_{d, \mathrm{SE}}$ are non-convex with respect to $P_{s}, P_{d}$, and $\beta$, so that directly solving problem (P2) introduces enormous computational complexity. To address this 
issue, the iterative optimization algorithm is adopted in this section. The basic idea is to first optimize the objective function over a portion of variables when the others are fixed and then joint optimize these variables by utilizing the separate optimization results. Specifically, we first optimize the problem (P2) over $P_{s}$ and $P_{d}$ with a fixed $\beta$ and then optimize the problem (P2) over $\beta$ with fixed $P_{s}$ and $P_{d}$. This process will be repeated until convergence.

\subsubsection{Optimization over $P_{s}$ and $P_{d}$ with a fixed $\beta$}

Given a fixed $\beta$, our objective is to seek the optimal transmission powers $P_{s}$ and $P_{d}$ to maximize $\eta_{d \text {, EE }}$ while satisfying the required constraints. Thus, given $\beta$, the subproblem (P2) can be rewritten as

$$
\begin{array}{ll}
\max _{P_{S}, P_{d}} & \frac{\eta_{d, S E}}{P_{d, \text { total }}} \\
\text { s.t. } & C 3: \eta_{d, S E} \geq \bar{\eta}_{d, S E} \\
& C 4: 0 \leq P_{s} \leq P_{s, \max } \\
& C 5: 0 \leq P_{d} \leq P_{d, \max }
\end{array}
$$

3.2.1.1 Theorem 2 For any given $\beta, \eta_{d, \mathrm{SE}}$ is a concave and strictly increasing function of $P_{s}$ and $P_{d}$ for $P_{s}, P_{d} \in$ $[0,+\infty)$.

\subsubsection{Proof Refer to Appendix 2.}

Although the constraints in the subproblem (P2-1) are convex according to Theorem 2, the objective function is still nonconvex. To solve this issue, we transform the objective function into a concave one by using the fractional programming technique developed in [22]. We define the maximum EE of the FDTW relay-assisted D2D link as $q_{d}^{*}$, which is given by

$$
q_{d}^{*}=\eta_{d, \mathrm{SE}}\left(P_{s}^{*}, P_{d}^{*}\right) / P_{d, \text { total }}\left(P_{s}^{*}, P_{d}^{*}\right)
$$

where $P_{s}^{*}$ and $P_{d}{ }^{*}$ are the optimal values at the maximum EE. The following theorem can be proved:

3.2.1.3 Theorem 3 The maximum EE $q_{d}^{*}$ is achieved if and only if $\eta_{d, \mathrm{SE}}\left(P_{s}, P_{d}\right)-q_{d}^{*} P_{d, \text { total }}\left(P_{s}, P_{d}\right)=\eta_{d, \mathrm{SE}}\left(P_{s}^{*}, P_{d}^{*}\right)$ $-q_{d}^{*} P_{d \text {,total }}\left(P_{s}^{*}, P_{d}^{*}\right)=0$ and for a given $q_{d}$, the transformed function in subtractive form is a concave function.

\subsubsection{Proof Refer to Appendix 3.}

Theorem 3 shows that the transformed problem with an objective function in subtractive form is equivalent to the non-convex problem (P2-1) in fractional form, i.e., they lead to the same optimum solution $P_{s}^{*}$ and $P_{d^{*}}$. Thus, there exists a nonfractional expression that is equal to a fractional function. Searching $q_{d}^{*}$ can be done by the Dinkelbach method that can converge to the optimal value at a superlinear rate [22]. Therefore, for any feasible $q_{d}$, the corresponding solution of subproblem (P2-1) can be obtained by solving the following equivalent transformed optimization subproblem (18):

$$
\begin{aligned}
& \max _{P_{s}, P_{d}} \eta_{d, \mathrm{SE}}\left(P_{s}, P_{d}\right)-q_{d} P_{d, \text { total }}\left(P_{s}, P_{d}\right) \\
& \text { s.t. } C_{3}, C_{4}, C_{5}
\end{aligned}
$$

The partial Lagrangian function associated with the subproblem (18) can be expressed as

$$
\begin{aligned}
L_{d, \mathrm{EE}}\left(P_{s}, P_{d}, \lambda_{1}\right) & =\eta_{d, \mathrm{SE}}\left(P_{s}, P_{d}\right)-q_{d} P_{d, \text { total }}\left(P_{s}, P_{d}\right) \\
& +\lambda_{1}\left(\eta_{d, \mathrm{SE}}-\bar{\eta}_{d, \mathrm{SE}}\right)
\end{aligned}
$$

where $\lambda_{1}$ is the Lagrangian multiplier of the inequality constraint $\mathrm{C} 1$ and is required to be nonnegative. Since the subproblem (18) is in a standard concave form with differentiable objective and constraint functions, the Karush-Kuhn-Tucker (KKT) conditions are used to find the optimum solutions. Therefore, the first-order derivative of $L_{d, \text { EE }}$ with respect to $P_{s}$ and $P_{d}$ is required to be zero, i.e.,

$$
\begin{aligned}
& \left(1+\lambda_{1}\right) \frac{\eta}{\eta P_{s}+\varphi_{s}}=q_{d} \ln 2\left(\xi+\beta G_{s r}\right) \\
& \left(1+\lambda_{1}\right) \frac{\eta}{\eta P_{d}+\varphi_{d}}=q_{d} \ln 2\left(\xi+\beta G_{r d}\right)
\end{aligned}
$$

In addition, since $\lambda_{1}$ is required to be a nonnegative one, the first-order derivative of $L_{d, \mathrm{EE}}$ with respect to $\lambda_{1}$ should satisfy $\lambda_{1} \frac{\partial L_{d, \mathrm{EE}}}{\partial \lambda_{1}}=0$, i.e.,

$$
\lambda_{1}\left(\frac{\eta}{\varphi_{d}} P_{d}+\frac{\eta}{\varphi_{s}} P_{s}+\frac{\eta^{2}}{\varphi_{d} \varphi_{s}} P_{d} P_{s}-\varpi\right)=0
$$

where $\eta=\beta G_{s r} G_{r d}, \quad \varphi_{s}=\beta P_{u} G_{r d} G_{u r}+\beta \rho_{r} G_{r d} G_{r r}+\beta G_{r d} \sigma^{2}$ $+P_{u} G_{u d}+\rho_{d} G_{d d}+\sigma^{2}$, and $\varphi_{d}=\beta P_{u} G_{s r} G_{u r}+\beta \rho_{r} G_{s r} G_{r r}$ $+\beta G_{s r} \sigma^{2}+P_{u} G_{u s}+\rho_{s} G_{s s}+\sigma^{2}, \varpi=2^{\bar{\eta}_{d, \mathrm{SE}}}-1$.

According to formula (20) and (21), we can obtain

$$
\begin{aligned}
& P_{s}^{*}=\left[\frac{1+\lambda_{1}}{q_{d} \ln 2\left(\xi+\beta G_{s r}\right)}-\frac{\varphi_{s}}{\eta}\right]^{+} \\
& P_{d}^{*}=\left[\frac{1+\lambda_{1}}{q_{d} \ln 2\left(\xi+\beta G_{r d}\right)}-\frac{\varphi_{d}}{\eta}\right]^{+}
\end{aligned}
$$

where $[x]^{+}$denotes $\max (0, x)$, which guarantees $P_{s} \geq 0$ and $P_{d} \geq 0$.

Notice that the optimal transmission power terms of SUE and DUE given by (23) and (24) are similar to traditional water-filling solutions, where the heights of the 
pool are defined as $\varphi_{s} / \eta$ and $\varphi_{d} / \eta$, and the water level is partially determined by $\lambda_{1}$ and $q_{d}$. Since both $P_{s}$ and $P_{d}$ involve the Lagrangian multiplier $\lambda_{1}$ and the KKT conditions of (22) require $\lambda_{1}$ to be nonnegative, we derive $\lambda_{1}$ in the following two cases.

Case 1: $\lambda_{1}>0$

In this case, according to formula (22), we have $\left(\eta / \varphi_{d}\right) P_{d}$ $+\left(\eta / \varphi_{s}\right) P_{s}+\left(\eta^{2} / \varphi_{d} \varphi_{s}\right) P_{d} P_{s}-\varpi=0$. After substituting (23) and (24), we have $\left(\lambda_{1}+1\right)^{2} S_{1}-S_{2}=0$, where $S_{1}=\eta^{2} /$ $(\ln 2)^{2} q_{d}^{2} \varphi_{d} \varphi_{s}\left(\xi+\beta G_{s r}\right)\left(\xi+\beta G_{r d}\right)$ and $S_{2}=\varpi+1$. Since $\lambda_{1}>0$, we retain the positive root, i.e., $\lambda_{1}^{*}=\sqrt{S_{2} / S_{1}}-1$. Substituting this positive root back to (23) and (24) yields the optimal solution of the subproblem (18) as

$$
\begin{aligned}
& P_{s}^{*}=\left[\frac{\sqrt{S_{2}}}{q_{d} \ln 2 \sqrt{S_{1}}\left(\xi+\beta G_{s r}\right)}-\frac{\varphi_{s}}{\eta}\right]^{+} \\
& P_{d}^{*}=\left[\frac{\sqrt{S_{2}}}{q_{d} \ln 2 \sqrt{S_{1}}\left(\xi+\beta G_{r d}\right)}-\frac{\varphi_{d}}{\eta}\right]^{+}
\end{aligned}
$$

Case 2: $\lambda_{1}=0$

In this case, the condition (22) always holds true. Hence, we have

$$
P_{s}^{*}=\left[\frac{1}{q_{d} \ln 2\left(\xi+\beta G_{s r}\right)}-\frac{\varphi_{s}}{\eta}\right]^{+}
$$

$$
P_{d}^{*}=\left[\frac{1}{q_{d} \ln 2\left(\xi+\beta G_{r d}\right)}-\frac{\varphi_{d}}{\eta}\right]^{+}
$$

Note that the solutions of case 2 may break the SE constraint. If this happens, the solution (25), (26) should be adopted. Otherwise, both solutions (25), (26) and (27), (28) are feasible, and the one leading to greater EE is the final solution. Considering the transmission power constraints, the final solution can be expressed as $\hat{P}_{s}=\min$ $\left(P_{d, \text { max }}, P_{s}^{*}\right)$ and $\hat{P}_{d}=\min \left(P_{d, \max }, P_{d}^{*}\right)$. Since the transformed problem (P2-1) is convex with a given $q_{d}$, the optimal EE can be obtained with a given $\beta$ by the Dinkelbach method. According to the above analysis, the detailed procedure of the Dinkelbach method is listed in Table 1.

\subsubsection{Optimization over $\beta$ with fixed $P_{s}$ and $P_{d}$}

In this subproblem, our objective is to maximize $\eta_{d, \mathrm{EE}}$ while satisfying the minimum required $\eta_{d, \mathrm{SE}}$ and the constraint of $\beta$, which can be formulated as

$$
\begin{gathered}
\max _{\beta} \eta_{d, E E} \\
\text { s.t. C } 3, \mathrm{C} 6
\end{gathered}
$$

It is easy to prove that $\eta_{d, \mathrm{SE}}$ is monotonically increasing function of $\beta \in[0,+\infty)$, and according to the restricted conditions of $\eta_{d, \mathrm{SE}}$ (i.e., $\eta_{d, S E} \geq 0, \eta_{d, S E} \geq \bar{\eta}_{d, S E}$

Table 1 Dinkelbach method

1) Initialize the maximum value $q_{d}^{(0)}=q_{d}^{0}, q_{d}^{(1)}=q_{d}^{1}$, the iteration index $i \leftarrow 1$, convergence index $\Delta \leftarrow 10^{-5}$, maximum number of iterations $N_{\max }^{i} \leftarrow 10$.

2) while $\left|q_{d}^{(i)}-q_{d}^{(i-1)}\right| \leq \Delta$ and $i \leq N_{\max }^{i}$ do.

a) Solve the subproblem (18): $\left(\hat{P}_{s}, \hat{P}_{d}\right)=\operatorname{argmax}_{P_{s}, P_{d}} \eta_{d, S E}\left(P_{s}, P_{d}\right)-q_{d}^{(i)} P_{d, \text { total }}\left(P_{s}, P_{d}\right)$,

b) Update the maximum value of $i-1$-th $q_{d}^{(i-1)} \leftarrow q_{d}^{(i)}$,

c) Update the iteration index $i \leftarrow i+1$,

d) Calculate the corresponding maximum value of $i$-th $q_{d}^{(i)}=\frac{\eta_{d, S E}\left(\hat{P}_{s}, \hat{P}_{d}\right)}{P_{d, \text { total }}\left(\hat{P}_{s}, \hat{P}_{d}\right)}$.

\section{3) end while.}

4) Output current $\hat{P}_{s}, \hat{P}_{d}$ and $q_{d}^{(l)}$ as $q_{d}^{*}$ respectively. 
), we have $\beta \in\left\{\beta \mid \beta \in\left[-\infty, \breve{\beta^{\prime}}\right] \cup[\widetilde{\beta},+\infty]\right\}$, where $\bar{\beta}^{\prime}=\left(-b-\sqrt{b^{2}-4 a c}\right) / 2 a, \bar{\beta}=\left(-b+\sqrt{b^{2}-4 a c}\right) / 2 a, a=$ $c_{2} c_{3}+c_{1} c_{4}+c_{1} c_{3}-\varpi c_{2} c_{4}, b=c_{3} \sigma_{1}+c_{1} \sigma_{2}-\varpi c_{2} \sigma_{2}-\varpi c_{4} \sigma_{1}$, and $c=-\varpi \sigma_{1} \sigma_{2}$. Since $\beta \in[0, \bar{\beta}]$ and $\beta \in\left\{\beta \mid \beta \in\left[-\infty, \widetilde{\beta^{\prime}}\right] U\right.$ $[\breve{\beta},+\infty]\}$, the feasible region of the subproblem (P2-2) can be rewritten as $\beta \in[\bar{\beta}, \bar{\beta}]$.

3.2.2.1 Theorem 4 Given $P_{s}, P_{d} \in[0,+\infty), \eta_{d, \mathrm{EE}}$ is strictly quasiconcave in $\beta$ for $\beta \in[0,+\infty)$.

\subsubsection{Proof Refer to Appendix 4.}

From Theorem 4, similar to the optimization problem of cellular link, there are only three cases for the curve $\eta_{d, \mathrm{EE}}$ vs. $\beta$ for $\beta \in[\bar{\beta}, \bar{\beta}]$.

Case 1: $\eta_{d, \mathrm{EE}}$ strictly increases with $\beta$ for $\beta \in[\bar{\beta}, \bar{\beta}]$, if $\left.\frac{d \eta_{d, \mathrm{EE}}}{d \beta}\right|_{\beta=\bar{\beta}} \geq 0$, where $\frac{d \eta_{d, \mathrm{EE}}}{d \beta}$ is given by (42) in
Appendix 4. In this case, the optimal solution to the subproblem (P2-2) is achieved at $\hat{\beta}=\bar{\beta}$.

Case 2: $\eta_{d, \mathrm{EE}}$ strictly decreases with $\beta$ for $\beta \in[\bar{\beta}, \bar{\beta}]$, if $\left.\frac{d \eta_{d, \mathrm{EE}}}{d \beta}\right|_{\beta=\breve{\beta}} \leq 0$. The optimal solution to the subproblem (P2-2) is achieved at $\hat{\beta}=\breve{\beta}$.

Case 3: $\eta_{d, \mathrm{EE}}$ first strictly increases and then strictly decreases with $\beta$ for $\beta \in[\bar{\beta}, \bar{\beta}]$ if $\left.\frac{d \eta_{d \mathrm{EE}}}{d \beta}\right|_{\beta=\bar{\beta}}<0$ and $\left.\frac{d \eta_{d, \mathrm{EE}}}{d \beta}\right|_{\beta=\bar{\beta}}>0$. The optimal solution to the subproblem (P2-2) is achieved at $\hat{\beta}=\beta^{*}$, where $\beta^{*}$ is the point at which $\eta_{d, \mathrm{EE}}$ reaches its maximum for $\beta \in[0,+\infty)$ and is obtained by solving the equation $\frac{d \eta_{d \text { EE }}}{d \beta}=0$, as shown in Appendix 4 . The exact expression of $\beta^{*}$ is not easily obtained and can be found by the numerical methods, such as the bisection method.

\subsubsection{Iterative optimization algorithm for the FDTW relay-assisted D2D link}

According to the previous analysis, by combining the solution processes in Sections 3.2.1 and 3.2.2, the maximum EE of the FDTW relay-assisted D2D link

Table $\mathbf{2}$ Iterative optimization algorithm

1) Initialize the transmission powers of SUE and DUE $\hat{P}_{s}^{(0)}=P_{s}^{0}, \hat{P}_{d}^{(0)}=P_{d}^{0}$, the iteration index $l \leftarrow 1$, convergence index $\Delta \leftarrow 10^{-5}$, maximum number of iterations $N_{\max }^{l} \leftarrow 10$, calculate the corresponding EE $\eta_{d, E E}^{*(0)}$ by formula (14) and also set $\eta_{d, E E}^{*(1)}=\eta_{d, E E}^{*(0)}+\varepsilon(\varepsilon>\Delta)$.

2) while $\left|\eta_{d, E E}^{*(l)}-\eta_{d, E E}^{*(l-1)}\right| \leq \Delta$ and $l \leq N_{\max }^{l}$ do.

a) Execute the method list in Table I to optimize over $P_{s}$ and $P_{d}$ for a fixed $\hat{\beta}^{(l+1)}$ in $(\mathrm{P} 2-1) \rightarrow \hat{P}_{s}^{(l+1)}$, $\hat{P}_{d}^{(l+1)}$,

b) Optimize over $\beta$ for fixed $\hat{P}_{s}^{(l+1)}$ and $\hat{P}_{d}^{(l+1)}$ in $(\mathrm{P} 2-2) \rightarrow \hat{\beta}^{(l+1)}$,

c) Calculate the corresponding SE $\eta_{d, S E}^{*(l+1)}$ and EE $\eta_{d, E E}^{*(l+1)}$ by (9) and (14), respectively,

d) Update the iteration index $l \leftarrow l+1$,

\section{3) end while.}

4) Output current $\eta_{d, S E}^{*}$ and $\eta_{d, E E}^{*}$. 
can be achieved. The basic idea is that the subproblem (P2-1) and the subproblem (P2-2) are solved repeated alternately by letting the output of one optimization be the input of the other to reach the solution of the primal problem (P2). The detailed procedure of the iterative optimization algorithm is listed in Table 2.

\subsection{Two-tier alternative iteration optimization algorithm}

In the formulated EE optimization problem, due to the existence of interference between cellular and D2D users, the best response of D2D link depends of cellular link; conversely, the best response of cellular link depends of D2D link. Therefore, by combining the solution processes in Section 3.1 and 3.2, the maximum EE of the whole system can be achieved. The basic idea is that the subproblem (P1) and the subproblem (P2) can be repeated alternately by letting the output of one optimization be the input of the other to reach the solution of the primal problem (16). For clarity, the detailed procedure of the twotier alternative iteration optimization algorithm is listed in Table 3, the first-tier iteration optimization indicates the D2D link optimization problem, and the second-tier iteration optimization indicates the primal optimization problem.

Firstly, under the given initial transmission power of CUE, to solve the EE optimization problem of D2D link, the algorithm list in Table 2 was used. Then, according to the obtained optimal power values and amplification gain value of D2D link, to solve the cellular link EE optimization problem, the bisection method was used. Repeat this process until convergence is reached, i.e., $\left|\eta_{\mathrm{EE}}^{*(n)}-\eta_{\mathrm{EE}}^{*(n-1)}\right|$ is equal or smaller than a predefined threshold $\Delta$.

Table 3 Two-tier alternative iteration optimization algorithm

1) Initialize the transmission powers of CUE $\hat{P}_{u}^{(0)}=P_{u}^{0}$, the iteration index $n \leftarrow 1$, convergence index $\Delta \leftarrow 10^{-5}$, maximum number of iterations $N_{\max }^{n} \leftarrow 10$, calculate the corresponding EE $\eta_{E E}^{*(0)}$ by formula (15) and also set $\eta_{E E}^{*(1)}=\eta_{E E}^{*(0)}+\varepsilon_{1}\left(\varepsilon_{1}>\Delta\right)$.

2) while $\left|\eta_{E E}^{*(n)}-\eta_{E E}^{*(n-1)}\right| \leq \Delta$ and $n \leq N_{\max }^{n}$ do.

\section{if $\mathrm{D} 2 \mathrm{D}$ link then}

Execute the algorithm list in Table II to solve the subproblem in (P2) $\rightarrow \hat{P}_{s}^{(n+1)}, \hat{P}_{d}^{(n+1)}, \hat{\beta}^{(n+1)}$

end if

if cellular link then

Applying the bisection method to solve the subproblem in (P1) $\rightarrow \hat{P}_{u}^{(n+1)}$

end if

3) Calculate the corresponding SE $\eta_{S E}^{*(n+1)}$ and EE $\eta_{E E}^{*(n+1)}$ by (10) and (15), respectively.

4) Update the iteration index $n \leftarrow n+1$.

5) end while.

6) Output current $\eta_{S E}^{*}$ and $\eta_{E E}^{*}$. 


\section{Simulation results}

In this section, we evaluate the performance of the proposed algorithm by using the MATLAB tool. The maximum transmission powers of SUE, DUE, RUE, and CUE are set to $P_{s, \text { max }}=1 \mathrm{~W}, P_{d, \text { max }}=1 \mathrm{~W}, P_{r, \text { max }}$ $=1 \mathrm{~W}$ and $P_{u, \max }=1 \mathrm{~W}$, respectively. The minimum data rate requirements of SUE, DUE, and CUE are set to $R_{s, \text { min }}=1 \mathrm{bit} / \mathrm{s} / \mathrm{Hz}, \quad R_{d, \text { min }}=1 \mathrm{bit} / \mathrm{s} / \mathrm{Hz}$, and $R_{u, \min }=1 \mathrm{bit} / \mathrm{s} / \mathrm{Hz}$, respectively. The circuit power consumption is set to $P_{c}=0.1 \mathrm{~W}$, and the power amplifier efficiency of users is set to $1 / \xi=1 / 0.35$. The power consumed by active DC in FD mode is set to $0.1 \mathrm{~W}$. The parameters of path-loss exponent and noise power are set to 4 and $-50 \mathrm{dBm}$, respectively. The bandwidth $W$ is normalized to 1 , i.e., $W=1 \mathrm{~Hz}$. These values of simulation parameters and channel gains are inspired by [18] and [26].

Figure 3 shows the locations of D2D users and cellular user. The BS is located at the center with coordinate $(0,0)$, the coordinates of SUE, DUE, and CUE are $(-50,-300),(50,-300)$, and $(0,100)$, respectively. We mainly consider the effect of RUE on system performance.

Figure 4 shows the convergence of the proposed algorithm in terms of average optimal EE. The RUE is located in the middle of SUE and DUE, i.e., $d_{s, r}=50 \mathrm{~m}$. It is shown that the proposed algorithm can achieve convergence quickly. Besides, we can find that the results obtained by the proposed optimization algorithm are very close to those obtained by the exclusive searching method, which indicates that the two-tier alternative iteration optimization algorithm can find a suboptimal solution.

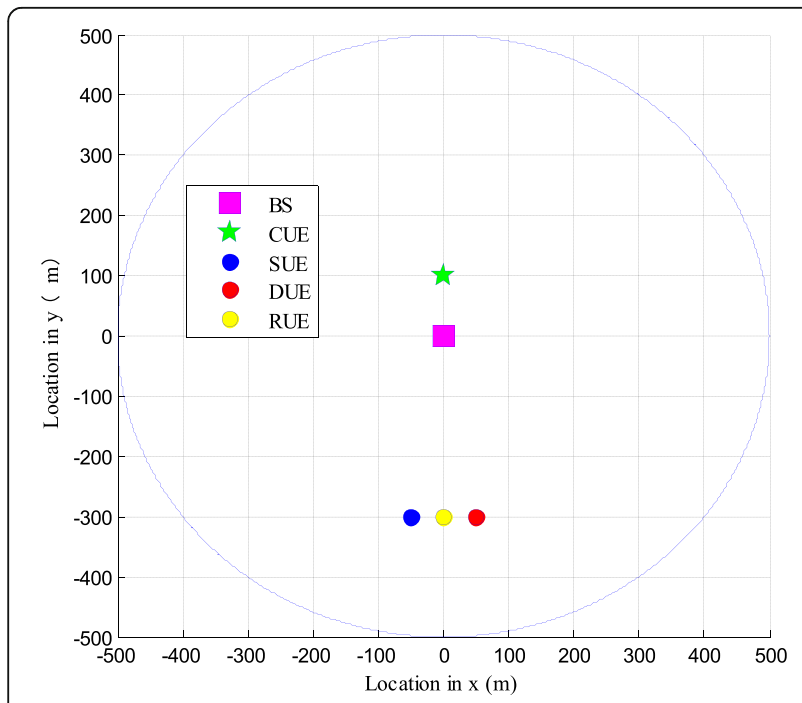

Fig. 3 The locations of D2D users and cellular user

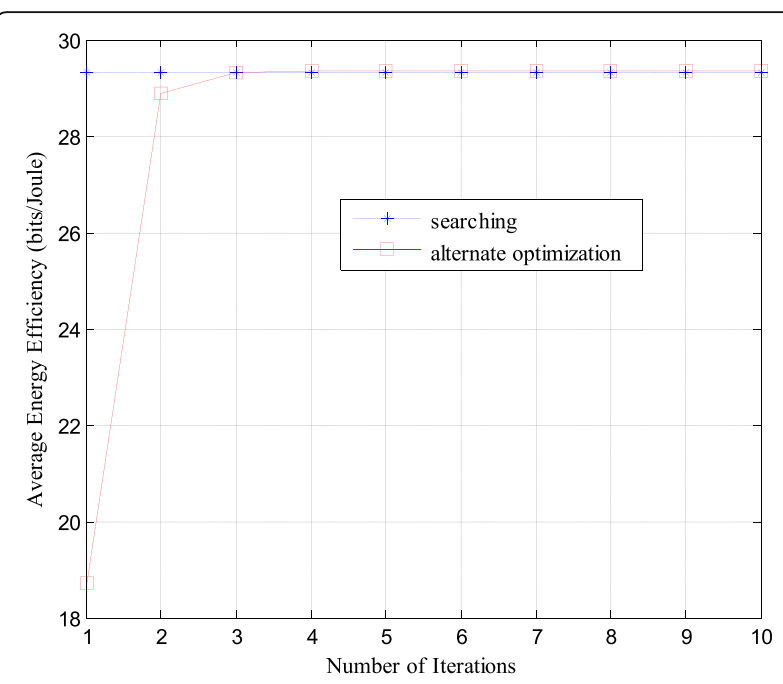

Fig. 4 Convergence of the proposed algorithm in terms of average optimal EE $(\rho=-3 \mathrm{~dB})$

Figures 5 and 6 show the comparison of the proposed algorithm and the exhaustive searching method when the power of SI is $\rho=-3 \mathrm{~dB}$. From the two figures, one can observe that average optimal EE first increases and then decreases as $d_{s, r}$ increases and reaches its maximum value at $d_{s, r}=50 \mathrm{~m}$ (i.e., the RUE is located in the center between SUE and the DUE). For average optimal SE, it can satisfy the constraint, which also first increases and then decreases as $d_{s, r}$ increases. In addition, simulation results in Fig. 7 also show that the amplification gain $\beta$ reaches its peak value at $d_{s, r}=50 \mathrm{~m}$. Hence, according to the

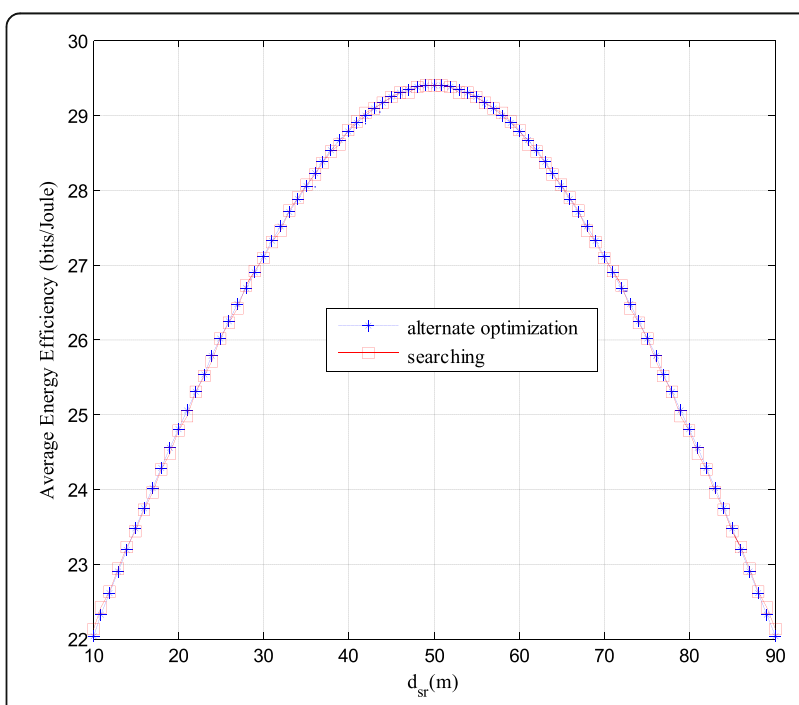

Fig. 5 Average optimal EE versus distance $d_{s, r}$ 


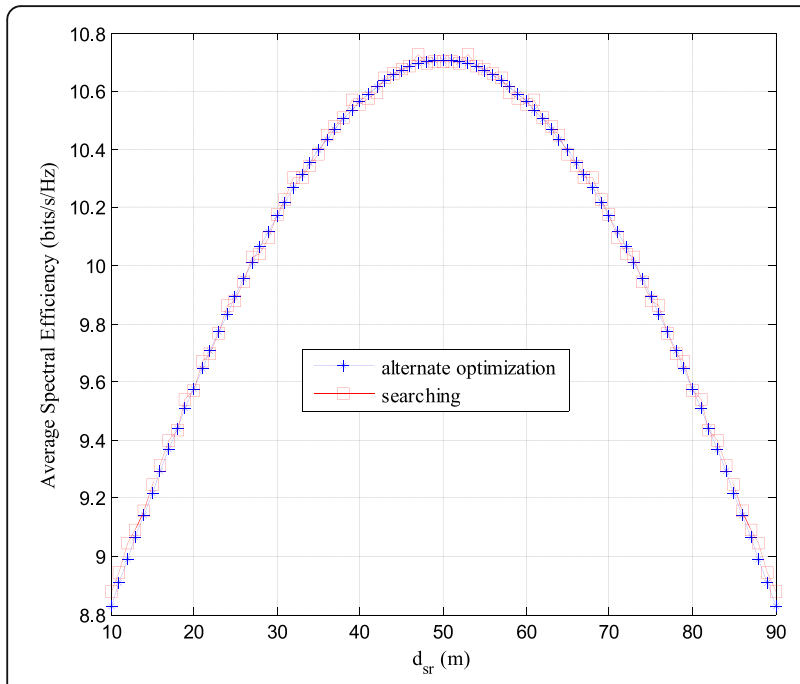

Fig. 6 Average optimal SE versus distance $d_{s, r}$

above analysis, when the D2D pair needs the relay to assist the transmission, the optimal transmission scheme is that the relay is located in the middle position of the D2D pair.

The exhaustive searching method needs to evaluate all the possible values of $P_{s}, P_{d}, \beta, P_{u}$, so it needs

$O\left(\frac{P_{s, \max } P_{d, \max } P_{u, \max }}{\varepsilon^{3}} \times \frac{\bar{\beta}-\bar{\beta}}{\varepsilon}\right) \quad$ steps to obtain the optimal value, where $\varepsilon$ is the searching accuracy. In the proposed algorithm, both the bisection method and the Dinkelbach method are adopted alternatively, which takes $O\left(M_{2}\left(M_{1}\left(M_{0}+\log _{2}((\bar{\beta}-\bar{\beta}) / \varepsilon)\right)+\log _{2}\left(P_{u, \max } / \varepsilon\right)\right)\right)$ steps, where $M_{0}, M_{1}$, and $M_{2}$ are the average iterative steps of the Dinkelbach method, first-tier and second-tier, respectively, and are both set to 10 in our simulation. Therefore, the proposed algorithm significantly reduces the computational complexity compared with the exhaustive search method.

Figures 8 and 9 show the average optimal EE and the average optimal SE versus different residual power of SI under the proposed two-tier alternative iteration optimization algorithm. The EE and the SE decrease with the increase of residual power of SI. Smaller residual power of SI leads to better performance of the system.

Figures 10 and 11 show the comparison of SE and $E E$ in terms of different residual power of SI. It can be observed in Fig. 10 that the SE obtained by FDTW relay-assisted D2D networks is higher than the SE obtained by HD counterpart, and this is mainly because the FD network can achieve higher SE compared with HD network. However, it also can be seen in Fig. 11 that the EE obtained by FDTW relay-assisted D2D networks is higher than the EE obtained by HD counterpart only when the residual power of SI is sufficiently small. Otherwise,

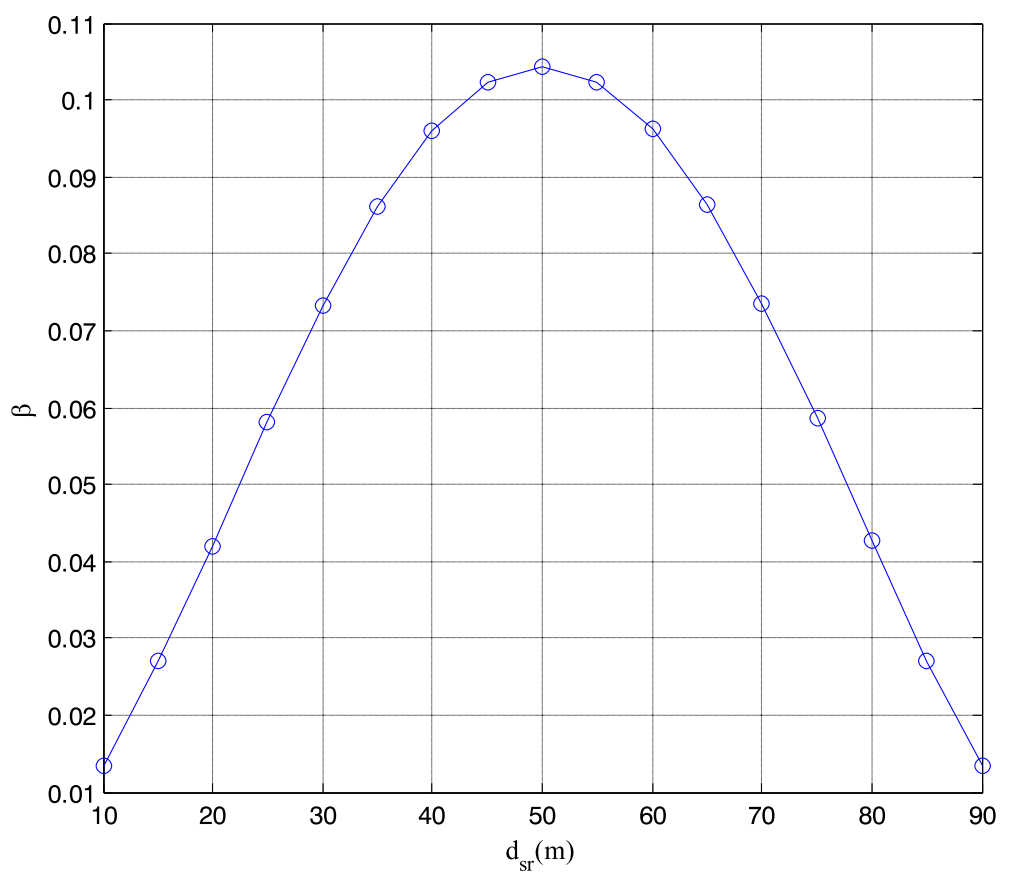

Fig. 7 Average $\beta$ versus distance $d_{s, r}$ 


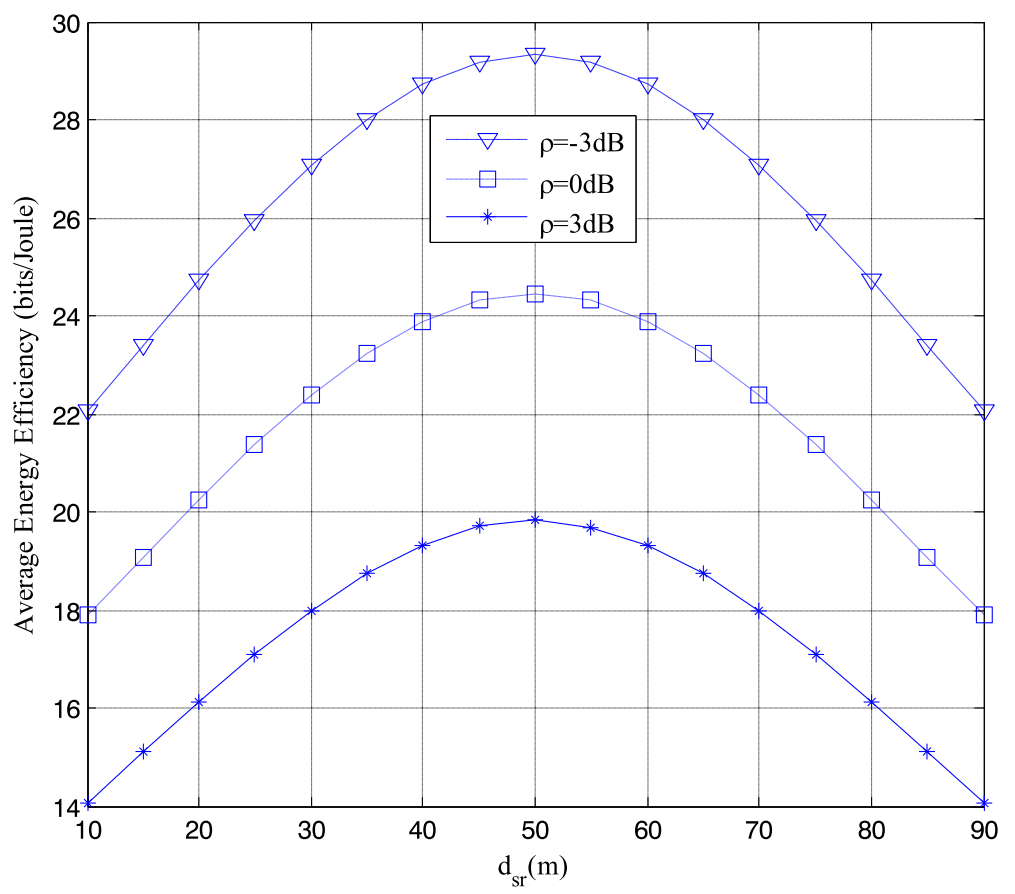

Fig. 8 Average optimal EE versus different residual power of SI

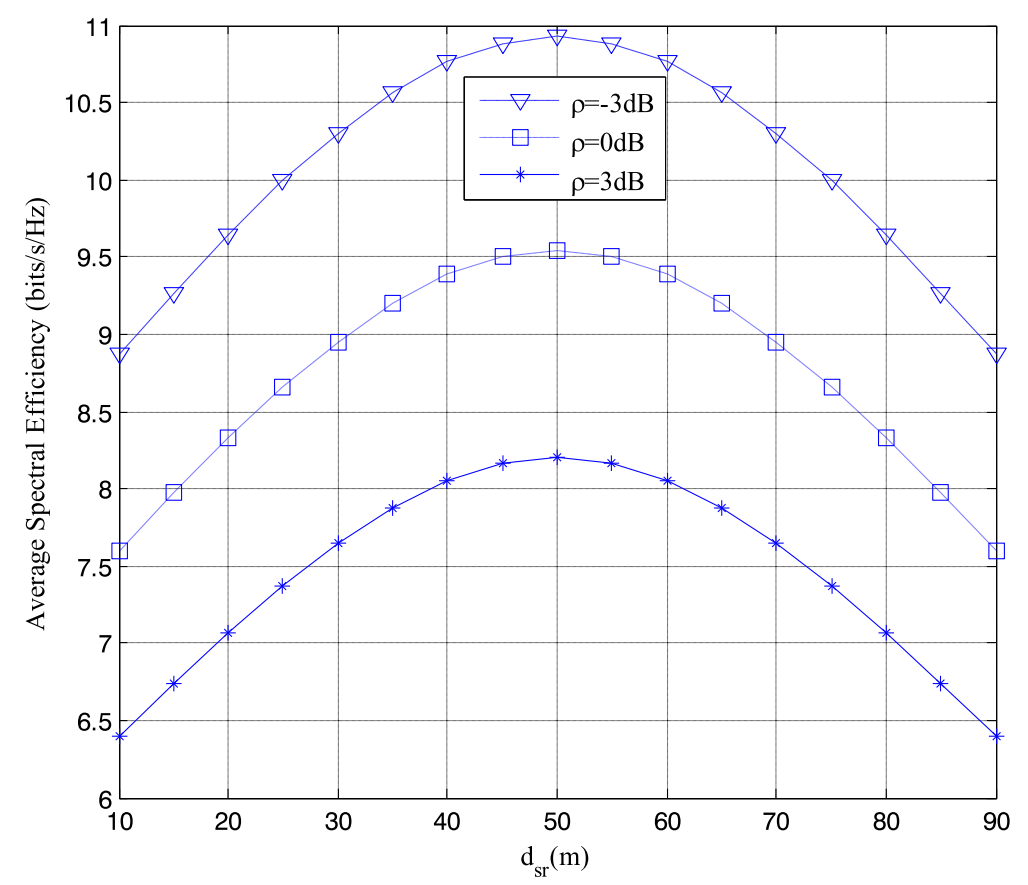

Fig. 9 Average optimal SE versus different residual power of SI 


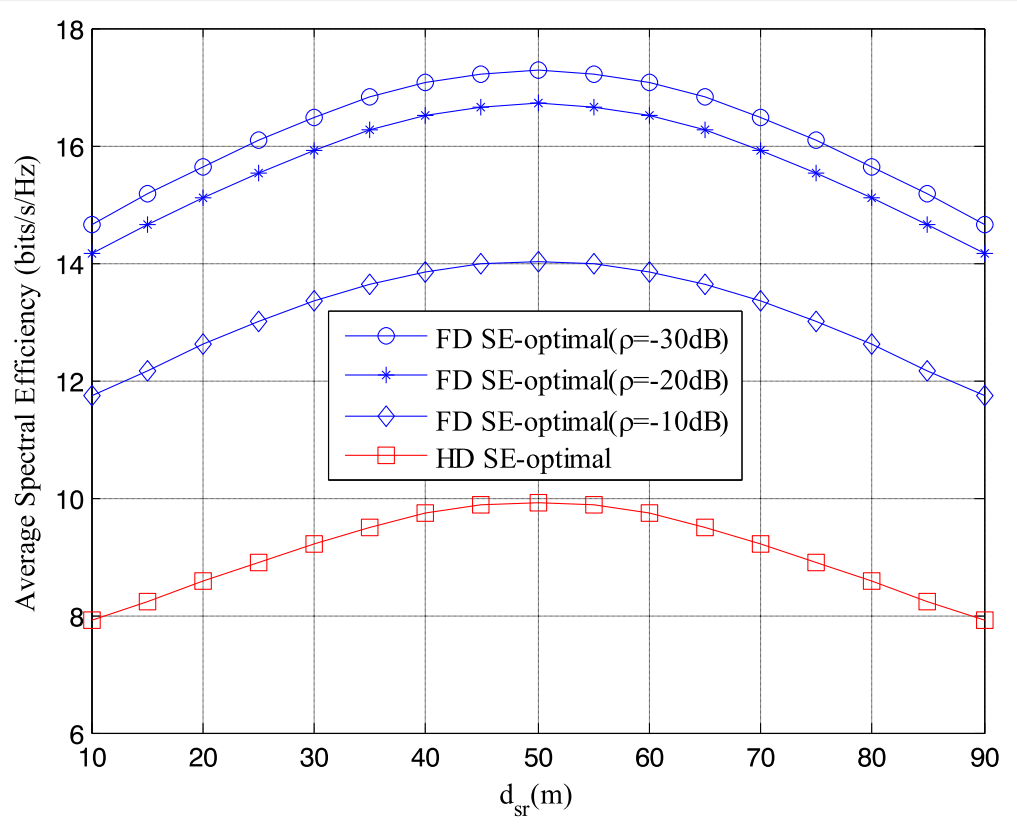

Fig. 10 Comparison of average optimal SE for FDTW and HDTW relay-assisted D2D networks

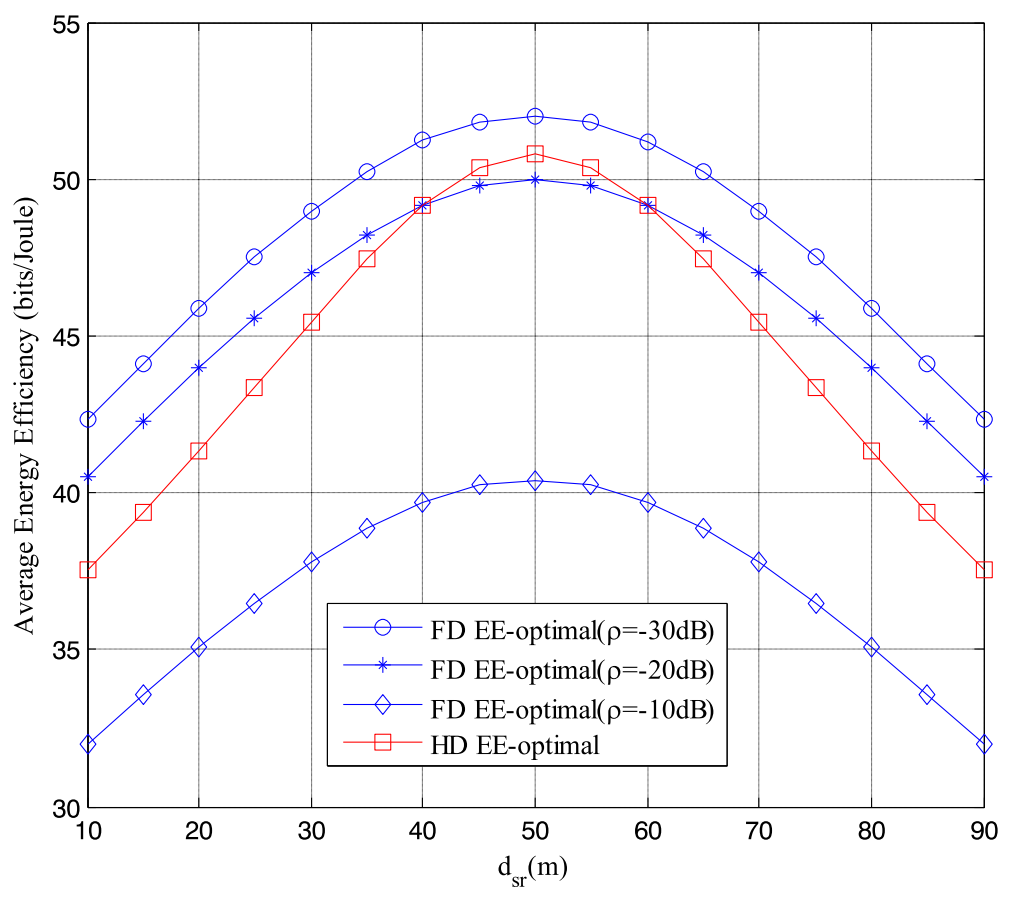

Fig. 11 Comparison of average optimal EE for FDTW and HDTW relay-assisted D2D networks 
the EE obtained by FDTW relay-assisted D2D networks is lower than the EE obtained by HD counterpart. These phenomena can be intuitively explained as follows. Although the FDTW relayassisted D2D network can achieve higher SE compared with the HD counterpart, the FD transmission mode has to overcome SI, which needs a larger power to transmit the signal. That is to say, it results in more power consumption than the HD network. Therefore, we can choose the appropriate transmission mode according to the SI cancelation techniques.

\section{Conclusions}

In this paper, the EE in FDTW relay-assisted D2D communications has been studied. By taking the SI at D2D users into consideration, the optimization problem in which the goal is to maximize EE while satisfying the SE requirement and the transmission power constraints was formulated. Since the optimization problem (16) is non-concave with respect to the variables $P_{u}, P_{s}, P_{d}$, and $\beta$, we decomposed it into two subproblems, i.e., cellular link optimization problem and D2D link optimization problem. As for the cellular link, the bisection method is used to seek the optimal solution. As for the D2D link, an iterative optimization algorithm was used to find the optimal solution. Then, a new two-tier alternative iteration optimization algorithm is proposed to solve the primal optimization problem. Simulation results show that the results of proposed algorithm are consistent with exhaustive searching results. The simulation results also indicate that smaller SI leads to higher SE and EE. Furthermore, the FDTW relay-assisted D2D network can achieve higher SE than the HDTW relayassisted D2D network. However, the EE obtained by FDTW relay-assisted D2D underlaying cellular networks is higher than the EE obtained by HD counterpart only when the residual power of SI is sufficiently small.

\section{Appendix 1}

\subsection{Proof of Theorem 1}

We take the first-order derivative of $\eta_{u \text {, EE }}$ with respect to $P_{u}$, which is represented by

$$
\frac{d \eta_{u, E E}}{d P_{u}}=\frac{f\left(P_{u}\right)}{\left(\xi P_{u}+P_{c}\right)^{2}}
$$

where

$$
\begin{aligned}
f\left(P_{u}\right)= & \frac{\left(\xi P_{u}+P_{c}\right)}{\ln 2\left(P_{u} G_{1}+G_{2}+P_{u} G_{u b}\right)\left(P_{u} G_{1}+G_{2}\right)} \\
& -\xi \log _{2}\left(1+\frac{P_{u} G_{u b}}{P_{u} G_{1}+G_{2}}\right)
\end{aligned}
$$

Then, the first-order derivative of $f\left(P_{u}\right)$ with respect to $P_{u}$ can be calculated as

$$
\begin{aligned}
& -\left(\xi P_{u}+P_{c}\right) G_{u b} G_{2}\left\{\left(G_{1}+G_{u b}\right)\left(P_{u} G_{1}+G_{2}\right)\right. \\
& \frac{d f\left(P_{u}\right)}{d P_{u}}=\frac{\left.+\left(P_{u} G_{1}+G_{2}+P_{u} G_{u b}\right) G_{1}\right\}}{\ln 2\left(P_{u} G_{1}+G_{2}+P_{u} G_{u b}\right)^{2}\left(P_{u} G_{1}+G_{2}\right)^{2}}<0
\end{aligned}
$$

Therefore, we have $f(+\infty)<f\left(P_{u}\right)<f(0), \quad \forall P_{u} \in[0$, $+\infty)$. Since $\lim _{P_{u} \rightarrow+\infty} f\left(P_{u}\right)=-\xi \log _{2}\left(G_{u b} / G_{1}\right)<0$ and $\lim _{P u \rightarrow 0} f\left(P_{u}\right)=\left(P_{c} / \ln 2\right)\left(G_{u b} / G_{2}\right)>0$, there exists a single value of $P_{u}$, which is denoted as $P_{u}{ }^{*}$, so that $f\left(P_{u}^{*}\right)=0$. It is obvious that the denominator of (29) is positive, and we have $d \eta_{u, E E} / d P_{u}>0$ when $P_{u}<P_{u}{ }^{*}$ and $d \eta_{u, E E} / d P_{u}<0$ when $P_{u}>P_{u}{ }^{*}$. It means that $\eta_{u, \mathrm{EE}}$ firstly increases and then decreases when $P_{u}$ in-

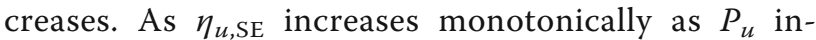
creases, $\eta_{u, S E}$ is a concave function of $P_{u}$. $P_{u \text {,total }}$ is an affine function of $P_{u}$. Therefore, $\eta_{u, \text { EE }}$ is quasiconcave in $P_{u}$ for $P_{u} \in[0,+\infty)$ [18]. Hence, the proof of Theorem 1 is complete.

\section{Appendix 2}

\subsection{Proof of Theorem 2}

We take the first-order partial derivatives of $\gamma_{s}$ and $\gamma_{d}$ with respect to $P_{s}$ and $P_{d}$, respectively, which are represented by

$$
\begin{aligned}
\frac{\partial \gamma_{s}}{\partial P_{d}} & =\frac{\beta G_{s r} G_{r d}}{\beta P_{u} G_{s r} G_{u r}+\beta \rho_{r} G_{s r} G_{r r}+\beta G_{s r} \sigma^{2}+P_{u} G_{u s}+\rho_{r} G_{s s}+\sigma^{2}} \\
\frac{\partial \gamma_{s}}{\partial P_{s}} & =0
\end{aligned}
$$$$
\frac{\partial \gamma_{d}}{\partial P_{s}}=\frac{\beta G_{s r} G_{r d}}{\beta P_{u} G_{r d} G_{u r}+\beta \rho_{r} G_{r d} G_{r r}+\beta G_{r d} \sigma^{2}+P_{u} G_{u d}+\rho_{r} G_{d d}+\sigma^{2}}
$$$$
\frac{\partial \gamma_{d}}{\partial P_{d}}=0
$$

Then, according to the formula (9), the first and the second partial derivatives of $\eta_{d, S E}$ with respect to $P_{s}$ and $P_{d}$ can be calculated as 


$$
\frac{\partial \eta_{d, S E}}{\partial P_{s}}=\frac{1}{\ln 2}\left(\frac{\beta G_{s r} G_{r d}}{\beta P_{u} G_{r d} G_{u r}+\beta \rho_{r} G_{r d} G_{r r}+\beta G_{r d} \sigma^{2}+P_{u} G_{u d}+\rho_{d} G_{d d}+\sigma^{2}+\beta P_{s} G_{s r} G_{r d}}\right)>0
$$

$$
\frac{\partial \eta_{d, S E}}{\partial P_{d}}=\frac{1}{\ln 2}\left(\frac{\beta G_{s r} G_{r d}}{\beta P_{u} G_{s r} G_{u r}+\beta \rho_{r} G_{s r} G_{r r}+\beta G_{s r} \sigma^{2}+P_{u} G_{u s}+\rho_{s} G_{s s}+\sigma^{2}+\beta P_{d} G_{s r} G_{r d}}\right)>0
$$

$$
\frac{\partial^{2} \eta_{d, S E}}{\partial P_{s}^{2}}=\frac{-1}{\ln 2}\left(\frac{\beta G_{s r} G_{r d}}{\beta P_{u} G_{r d} G_{u r}+\beta \rho_{r} G_{r d} G_{r r}+\beta G_{r d} \sigma^{2}+P_{u} G_{u d}+\rho_{d} G_{d d}+\sigma^{2}+\beta P_{s} G_{s r} G_{r d}}\right)^{2}<0
$$

$$
\begin{aligned}
& \frac{\partial^{2} \eta_{d, S E}}{\partial P_{d}^{2}}=\frac{-1}{\ln 2}\left(\frac{\beta G_{s r} G_{r d}}{\beta P_{u} G_{s r} G_{u r}+\beta \rho_{r} G_{s r} G_{r r}+\beta G_{s r} \sigma^{2}+P_{u} G_{u s}+\rho_{s} G_{s s}+\sigma^{2}+\beta P_{d} G_{s r} G_{r d}}\right)^{2}<0 \\
& \frac{\partial^{2} \eta_{d, S E}}{\partial P_{d} \partial P_{s}}=\frac{\partial^{2} \eta_{d, S E}}{\partial P_{s} \partial P_{d}}=0 \\
& \eta_{d, \mathrm{SE}}\left(P_{s}, P_{d}\right)-q_{d}^{*} P_{d, \text { total }}\left(P_{s}, P_{d}\right) \leq \eta_{d, \mathrm{SE}}\left(P_{s}^{*}, P_{d}^{*}\right) \\
& -q_{d}^{*} P_{d, \text { total }}\left(P_{s}^{*}, P_{d}^{*}\right)=0
\end{aligned}
$$

$$
\frac{\partial^{2} \eta_{d, S E}}{\partial P_{s}^{2}} \frac{\partial^{2} \eta_{d, S E}}{\partial P_{d}^{2}}-\frac{\partial^{2} \eta_{d, S E}}{\partial P_{s} \partial P_{d}} \frac{\partial^{2} \eta_{d, S E}}{\partial P_{d} \partial P_{s}}>0
$$

From (30) to (33), we can obtain that the Hessian matrix of $\eta_{d, S E}$ is negatively definite. Thus, $\eta_{d, S E}$ is a concave function for $P_{s}, P_{d} \in[0,+\infty)$. Hence, the proof of Theorem 2 is complete.

\section{Appendix 3}

\subsection{Proof of Theorem 3}

Define $F\left(q_{d}\right)=\eta_{d, \mathrm{SE}}\left(P_{s}, P_{d}\right)-q_{d} P_{d \text {,total }}\left(P_{s}, P_{d}\right)$. We prove the equivalence and convexity, respectively.

The proof of equivalence is divided into two parts: necessity and sufficiency. Firstly, we prove the necessity. Suppose that the optimal EE of the subproblem (P2-1) is $q_{d}^{*}=\eta_{d, \mathrm{SE}}\left(P_{s}^{*}, P_{d}^{*}\right) / P_{d, \text { total }}\left(P_{s}^{*}, P_{d}^{*}\right)$. Then, for any feasible region of $P_{s}$ and $P_{d}$, we have

$$
q_{d}^{*}=\frac{\eta_{d, \mathrm{SE}}\left(P_{s}^{*}, P_{d}^{*}\right)}{P_{d, \text { total }}\left(P_{s}^{*}, P_{d}^{*}\right)} \geq \frac{\eta_{d, \mathrm{SE}}\left(P_{s}, P_{d}\right)}{P_{d, \text { total }}\left(P_{s}, P_{d}\right)}
$$

By rearranging (38), we obtain

Hence, the maximum value of $\eta_{d, \mathrm{SE}}\left(P_{s}, P_{d}\right)-q_{d}^{*} P_{d \text {,total }}$ $\left(P_{s}, P_{d}\right)$ is equal to zero and can only be achieved at $P_{s}=P_{s}^{*}, P_{d}=P_{d}^{*}$. This completes the necessity proof.

Next, we turn to the sufficiency proof. Assume that $\quad \eta_{d, \mathrm{SE}}\left(P_{s}, P_{d}\right)-q_{d}^{*} P_{d, \text { total }}\left(P_{s}, P_{d}\right)=\eta_{d, \mathrm{SE}}\left(P_{s}^{*}, P_{d}^{*}\right)-q_{d}^{*}$ $P_{d, \text { total }}\left(P_{s}^{*}, P_{d}^{*}\right)=0$. Then, for given any feasible region of $P_{s}$ and $P_{d}$, we have

$$
\begin{gathered}
\eta_{d, \mathrm{SE}}\left(P_{s}, P_{d}\right)-q_{d}^{*} P_{d, \text { total }}\left(P_{s}, P_{d}\right) \leq \eta_{d, \mathrm{SE}}\left(P_{s}^{*}, P_{d}^{*}\right) \\
-q_{d}^{*} P_{d, \text { total }}\left(P_{s}^{*}, P_{d}^{*}\right)=0
\end{gathered}
$$

By rearranging (40), we have

$$
\frac{\eta_{d, \mathrm{SE}}\left(P_{s}, P_{d}\right)}{P_{d, \text { total }}\left(P_{s}, P_{d}\right)} \leq \frac{\eta_{d, \mathrm{SE}}\left(P_{s}^{*}, P_{d}^{*}\right)}{P_{d, \text { total }}\left(P_{s}^{*}, P_{d}^{*}\right)}=q_{d}^{*}
$$

Thus, the maximum value of EE can be obtained at $P_{s}=P_{s}^{*}, P_{d}=P_{d}^{*}$, and this completes the sufficiency proof. Therefore, the optimal solution to the subproblem (P2-1) and the problem $\max _{P_{s}, P_{d}} F\left(q_{d}^{*}\right)=0$ are equivalent.

Now, we prove the convexity. For any given $q_{d}$, the transformed subtractive form can be written as $\eta_{d, \mathrm{SE}}\left(P_{s}\right.$, 
$\left.P_{d}\right)-q_{d} P_{d, \text { total }}\left(P_{s}, P_{d}\right)$. The first part of the subtractive form is $\eta_{d, S E}\left(P_{s}, P_{d}\right)$, which is a concave function for $P_{s}$, $P_{d} \in[0,+\infty)$ according to Theorem 2 . The second part is an affine function. Since the sum of a concave function and an affine function is also concave, this completes the convexity proof.

\section{Appendix 4}

\subsection{Proof of Theorem 4}

Firstly, we introduce the following equations and inequality:

$$
\begin{aligned}
c_{1} & =P_{d} G_{s r} G_{r d}, c_{2}=P_{u} G_{s r} G_{u r}+\rho_{r} G_{s r} G_{r r}+G_{s r} \sigma^{2}, \sigma_{1}=P_{u} G_{u s} \\
& +\rho_{s} G_{s s}+\sigma^{2} \\
c_{3} & =P_{s} G_{s r} G_{r d}, c_{4}=P_{u} G_{r d} G_{u r}+\rho_{r} G_{r d} G_{r r}+G_{r d} \sigma^{2}, \sigma_{2}=P_{u} G_{u d} \\
& +\rho_{d} G_{d d}+\sigma^{2} \\
a_{1} & =P_{s} G_{s r}+P_{d} G_{r d}+\rho_{r} G_{r r}+P_{u} G_{u r}+\sigma^{2}, a_{2}=\xi P_{s}+\xi P_{d}+3 P_{c} \\
k_{1} & =c_{2} c_{4}+c_{2} c_{3}+c_{1} c_{4}+c_{1} c_{3}, k_{2}=c_{2} \sigma_{2}+c_{1} \sigma_{2}+c_{3} \sigma_{1}+c_{4} \sigma_{1}, \\
k_{3} & =c_{2} c_{4}, k_{4}=c_{2} \sigma_{2}+\sigma_{1} c_{4} \\
k_{3}^{2} & -k_{1}^{2}<0, k_{3} k_{4}-k_{1} k_{2}<0,2 \sigma_{1} \sigma_{2}\left(k_{1}-k_{3}\right)+k_{4}^{2}-k_{2}^{2}<0
\end{aligned}
$$

We take the first-order derivatives of $\eta_{d, E E}$ with respect to $\beta$, which is given by

$$
\frac{d \eta_{d, E E}}{d \beta}=\frac{f(\beta)}{\left(a_{1} \beta+a_{2}\right)^{2}}
$$

where

$$
\begin{aligned}
f(\beta)= & \frac{\left(a_{1} \beta+a_{2}\right)}{\ln 2}\left(\frac{2 k_{1} \beta+k_{2}}{k_{1} \beta^{2}+k_{2} \beta+\sigma_{1} \sigma_{2}}-\frac{2 k_{3} \beta+k_{4}}{k_{3} \beta^{2}+k_{4} \beta+\sigma_{1} \sigma_{2}}\right) \\
& -a_{1} \log _{2} \frac{k_{1} \beta^{2}+k_{2} \beta+\sigma_{1} \sigma_{2}}{k_{3} \beta^{2}+k_{4} \beta+\sigma_{1} \sigma_{2}}
\end{aligned}
$$

Then, the first-order derivatives of $f(\beta)$ with respect to $\beta$ can be calculated as

$$
\begin{aligned}
\frac{d f(\beta)}{d \beta}= & \frac{\left(a_{1} \beta+a_{2}\right)}{\ln 2}\left(\frac{2 k_{1}\left(k_{1} \beta^{2}+k_{2} \beta+\sigma_{1} \sigma_{2}\right)-\left(2 k_{1} \beta+k_{2}\right)^{2}}{\left(k_{1} \beta^{2}+k_{2} \beta+\sigma_{1} \sigma_{2}\right)^{2}}\right. \\
& \left.-\frac{2 k_{3}\left(k_{3} \beta^{2}+k_{4} \beta+\sigma_{1} \sigma_{2}\right)-\left(2 k_{3} \beta+k_{4}\right)^{2}}{\left(k_{3} \beta^{2}+k_{4} \beta+\sigma_{1} \sigma_{2}\right)^{2}}\right) \\
& \leq \frac{\left(a_{1} \beta+a_{2}\right)}{\ln 2}\left(\begin{array}{c}
\frac{2\left(k_{3}^{2}-k_{1}^{2}\right) \beta^{2}+2\left(k_{3} k_{4}-k_{1} k_{2}\right) \beta}{+2 \sigma_{1} \sigma_{2}\left(k_{1}-k_{3}\right)+k_{4}^{2}-k_{2}^{2}} \\
\left(k_{3} \beta^{2}+k_{4} \beta+\sigma_{1} \sigma_{2}\right)^{2}
\end{array}\right) \\
& <0, \beta \in[0,+\infty)
\end{aligned}
$$

Therefore, we have $f(+\infty)<f(\beta)<f(0), \quad \forall \beta \in[0,+\infty)$. Since $\lim _{\beta \rightarrow+\infty} f(\beta)=-a_{1} \log _{2}\left(k_{1} / k_{3}\right)<0$ and $\lim _{\beta \rightarrow 0} f(\beta)$ $=\left(a_{2} / \ln 2\right)\left(\left(k_{2}-k_{4}\right) / \sigma_{1} \sigma_{2}\right)>0$, there exists a single value of $\beta$, which is denoted as $\beta^{*}$, so that $f\left(\beta^{*}\right)=0$. It is obvious that the denominator of (42) is positive, we have $d \eta_{d, E E} / d \beta>0$ when $\beta<\beta^{*}$ and $d \eta_{d, E E} / d \beta<0$ when $\beta>\beta^{*}$.
It means that $\eta_{d, E E}$ firstly increases and then decreases when $\beta$ increases. As $\eta_{d, \mathrm{SE}}$ increases monotonically as $\beta$ increases, $\eta_{d, \mathrm{SE}}$ is a concave function of $\beta . P_{d, \text { total }}$ is an affine function of $\beta$. Therefore, $\eta_{d, E E}$ is quasiconcave in $\beta$ for $\beta \in[0,+\infty)$ [18]. Hence, the proof of Theorem 4 is complete.

\section{Acknowledgements}

This work was supported by the National Natural Science Foundation of China $(61471135,61671165)$, the Guangxi Natural Science Foundation (2013GXNSFGA019004, 2015GXNSFBB139007), the Fund of Key Laboratory Cognitive Radio and Information Processing, Guilin University of Electronic Technology, China, and Guangxi Key Laboratory of Wireless Wideband Communication and Signal Processing (CRKL150104, CRKL160105), the Innovation Project of Guangxi Graduate Education (YCSZ2015144), and the Innovation Project of GUET Graduate Education (2016YJCX91).

\section{Competing interests}

The authors declare that they have no competing interests.

Received: 3 July 2016 Accepted: 6 September 2016

Published online: 17 September 2016

\section{References}

1. A Gupta, RK Jha, A survey of $5 \mathrm{G}$ network: architecture and emerging technologies. IEEE Access 3, 1206-1232 (2015)

2. P Demestichas, A Georgakopoulos, D Karvounas, K Tsagkaris, V Stavroulaki, J Lu, C Xiong, J Yao, 5G on the horizon: key challenges for the radio-access network. IEEE Veh Tech Maga 8(3), 47-53 (2013)

3. A Zakrzewska, S Ruepp, MS Berger, Towards converged $5 \mathrm{G}$ mobile networkschallenges and current trends. Proc. ITU Kaleidoscope Academic Conf, 2014, pp. 39-45

4. S Talwar, D Choudhury, K Dimou, E Aryafar, B Bangerter, K Stewart, Enabling technologies and architectures for $5 G$ wireless. Proc. IEEE Microwave Symp, 2014, pp. 1-4

5. N Reider, G Fodor, A distributed power control and mode selection algorithm for D2D communications. EURASIP J Wireless Commun Netw 2012(1), 1-25 (2012)

6. Wen, X. Zhu, Y. Lin, Z. Lin, X. Zhang, D. Yang, "Achievable transmission capacity of relay-assisted device-to-device (D2D) communication underlay cellular networks," in Proc. IEEE VTC Fall, pp. 1-5, Sept. 2013

7. Z Hasan, H Boostanimehr, VK Bhargava, Green cellular networks: a survey, some research issues and challenges. IEEE Commun Surv Tutorials 13(4), 524-540 (2011)

8. $\quad \mathrm{Y} \mathrm{Ni}, \mathrm{S}$ Jin, KK Wong, H Zhu, S Shao, Outage performances for device-todevice communication assisted by two-way amplify-and-forward relay protocol. Proc. IEEE Wireless Communications and Networking Conf, 2014, pp. 502-507

9. Y Zhao, Y Li, N Ge, Physical layer network coding aided two-way device-todevice communication underlaying cellular networks. Proc. IEEE Global Commun. Conf, 2015, pp. 1-6

10. G Liu, FR Yu, H Ji, VCM Leung, X Li, In-band full-duplex relaying: a survey, research issues and challenges. IEEE Commun Surv Tutorials 17(2), 500-524 (2015)

11. Z Zhang, K Long, AV Vasilakos, L Hanzo, Full-duplex wireless communications: challenges, solutions, and future research directions. Proc IEEE 104(7), 1369-1409 (2016)

12. L Song, $Y \mathrm{Li}$, Z Han, Resource allocation in full-duplex communications for future wireless networks. IEEE Wireless Commun 22(4), 88-96 (2015)

13. E Ahmed, AM Eltawil, Z Li, BA Cetiner, Full-duplex systems using multireconfigurable antennas. IEEE Trans Wireless Commun 14(11), 5971-5983 (2015)

14. E Ahmed, AM Eltawil, All-digital self-interference cancellation technique for full-duplex systems. IEEE Trans Wireless Commun 14(7), 3519-3532 (2015)

15. S Li, RD Murch, An investigation into baseband techniques for singlechannel full-duplex wireless communication systems. IEEE Trans Wireless Commun 13(9), 4794-4806 (2014)

16. YA Sambo, MZ Shakir, KA Qaraqe, E Serpedin, Energy efficiency improvements in HetNets by exploiting device-to-device communications. Proc. IEEE Signal Process. Conf., 2014, pp. 151-155 
17. M Sheng, $Y L i, X$ Wang, J Li, Y Shi, Energy efficiency and delay tradeoff in device-to-device communications underlaying cellular networks. IEEE J Sel Areas Commun 34(1), 92-106 (2016)

18. Z Zhou, M Dong, K Ota, J Wu, T Sato, Energy efficiency and spectral efficiency tradeoff in device-to-device (D2D) communications. IEEE Wireless Commun Lett 3(5), 485-488 (2014)

19. JMB da Silva, G Fodor, TF Maciel, Performance analysis of network-assisted two-hop D2D communications. Proc. IEEE Globecom Workshops, 2014, pp. 1050-1056

20. Y Cai, Y Ni, H Zhu, Performance for device-to-device communication with three-time-slot two-way amplify-and-forward relay protocol. China Commun 12(11), 1-11 (2015)

21. L Wei, R Hu, Y Qian, G Wu, Energy-efficiency and spectrum-efficiency of multi-hop device-to-device communications underlaying cellular networks. IEEE Trans Veh Tech 65(1), 367-380 (2015)

22. S Schaible, T Ibaraki, Fractional programming. Eur J Oper Res 12(4), 325-338 (1983)

23. M Duarte, C Dick, A Sabharwal, Experiment-driven characterization of fullduplex wireless systems. IEEE Trans Wireless Commun 11(12), 4296-4307 (2012)

24. L Song, Y Liao, K Bian, L Song, Z Han, Cross-layer protocol design for CSMA CD in full-duplex WiFi networks. IEEE Commun Lett 20(4), $792-795$ (2016)

25. D Bharadia, E McMilin, S Katti, Full duplex radios. ACM SIGCOMM Computer Commun Review 43(4), 375-386 (2013)

26. H. Chen, G. Li, J. Cai, "Spectral-energy efficiency tradeoff in full-duplex twoway relay networks," IEEE Syst. J., published online.

27. M Jain et al., Practical, real-time, full duplex wireless. Proc. ACM MobiCom, 2011, pp. 301-312

28. RHY Louie, Y Li, B Vucetic, Practical physical layer network coding for twoway relay channels: performance analysis and comparison. IEEE Trans Wireless Commun 9(2), 764-777 (2010)

29. Y Zhao, Y Li, H Zhang, N Ge, J Lu, Fundamental tradeoffs on energy-aware D2D communication underlaying cellular networks: a dynamic graph approach. IEEE J Sel Areas Commun 34(4), 864-882 (2016)

30. G Miao, N Himayat, GY Li, S Talwar, Distributed interference-aware energyefficient power optimization. IEEE Trans Wireless Commun 10(4), 1323-1333 (2011)

\section{Submit your manuscript to a SpringerOpen ${ }^{\circ}$ journal and benefit from:}

- Convenient online submission

- Rigorous peer review

- Immediate publication on acceptance

- Open access: articles freely available online

- High visibility within the field

- Retaining the copyright to your article 